\title{
Synthesis of 1,2,3-Triazolo Fused Allocolchicine Analogs via Intramolecular Oxidative Biaryl Coupling
}

\author{
Besir Krasniqi and Wim Dehaen * \\ Molecular Design and Synthesis, Department of Chemistry, KU Leuven, Celestijnenlaan 200F, \\ 3001 Leuven, Belgium
}

\section{Supporting information}

\section{Table of Contents}

General Considerations... S2

General procedure for the preparation of 1,2,3-triazole substrate S2

General Procedure Oxidative Coupling Reactions. S2

4-Nitrophenyl Azide. S2

Separation of atropoisomers of compound 7a by using Chiral HPLC S11

References. . $\mathrm{S} 11$

${ }^{1} \mathrm{H}$ and ${ }^{13} \mathrm{C}$ NMR spectra S13 


\section{General Considerations}

NMR spectra were recorded on a Bruker 400 avance (400 MHz) or a Bruker 300 and 600 Avance II+ (600 $\mathrm{MHz})$, and chemical shifts $(\delta)$ are reported part per million $(\mathrm{ppm})$ referenced to tetramethylsilane $\left({ }^{1} \mathrm{H}\right)$. Melting points were determined using a Reichert Thermovar apparatus. For column chromatography 70230 mesh silica 60 (E. M. Merck) was used as the stationary phase. Chemicals received from commercial sources were used without further purification. Reaction dry solvents were used as received from commercial sources. TLC were carried out on Kieselgel 60 F254 plates (Merck) and visualized with UVlamp $254 \mathrm{~nm}$.

Exact mass spectra were acquired with a quadrupole orthogonal acceleration time-of-flight mass spectrometer (Synapt G2 HDMS, Waters, Milford, MA). Samples were infused at $3 \mathrm{uL} / \mathrm{min}$ and spectra were obtained in positive (or: negative) ionization mode with a resolution of 15000 (FWHM) using leucine enkephalin as lock mass.

\section{General procedure for the preparation of 1,2,3-triazole substrate}

To a screw-capped reaction tube equipped with a magnetic stirring bar was added the ketone (1.0 equiv.), primary amine (1.3 equiv.), 4-nitrophenyl azide (1.0 equiv.) and $4 \AA$ molecular sieves ( $50 \mathrm{mg}$ ). The mixture was dissolved in dry toluene and stirred at $100{ }^{\circ} \mathrm{C}$ for 24 hours. The crude reaction mixture was then directly purified by column chromatography (silica gel) at first was used dichloromethane as eluent to remove 4nitroaniline formed during the reaction followed by using a mixture of Petroleum ether and ethyl acetate as eluent to afford the corresponding 1,2,3-triazole compound. ${ }^{1}$

\section{General Procedure Oxidative Coupling Reactions}

To a stirred solution of 1,2,3-triazole substrate (1.0 equiv.) in dry dichloromethane $(2.0 \mathrm{~mL})$ was added a solution of phenyliodine bis(trifluoroacetate) PIFA (1.1 equiv.) in dry dichloromethane $(2.0 \mathrm{~mL})$ and $\mathrm{BF}_{3} \cdot \mathrm{Et}_{2} \mathrm{O}$ (3.0 equiv.) at $-40{ }^{\circ} \mathrm{C}$ under argon atmosphere and the mixture was stirred for $3 \mathrm{~h}$. After confirmation that the starting material had been consumed via TLC the reaction was quenched with $\mathrm{NaHCO}_{3}$ solution and extracted with DCM. The crude was purified via column chromatography (silica gel) where as the eluent was used petroleum ether and ethyl acetate. In some cases, used preparative TLC was used for further purification.

\section{4-Nitrophenyl Azide}

To a $3 \mathrm{~L}$ flask equipped with mechanical stirrer, 4-nitroaniline $(28.0 \mathrm{~g}, 0.20 \mathrm{~mol})$ was suspended in a concentrated solution of $\mathrm{HCl}(300 \mathrm{~mL})$ then methanol $(60 \mathrm{~mL})$ was added and stirred for $2 \mathrm{~h}$ at r.t. Next the mixture was cooled at $0-5{ }^{\circ} \mathrm{C}$, if 4-nitroaniline still was not dissolved more concentrated $\mathrm{HCl}$ was added until a clear solution was obtained, then $\mathrm{NaNO}_{2}$ solution $(6 \mathrm{M}, 50 \mathrm{~mL})$ was added dropwise keeping the temperature below $5^{\circ} \mathrm{C}$ during the addition. The mixture was stirred at $0-5{ }^{\circ} \mathrm{C}$ for 30 minutes, after that a solution of $\mathrm{NaN}_{3}(4.1 \mathrm{M}, 80 \mathrm{~mL})$ was added dropwise over 30 minutes and the whole reaction mixture was stirred for an hour at room temperature (warning: the volume of the reaction mixture increased drastically due to $\mathrm{N}_{2}$ liberation therefore a large flask is needed to accommodate this foaming). The precipitate was filtered, washed with a saturated $\mathrm{NaHCO}_{3}$ solution and water, dissolved in diethyl ether, dried over $\mathrm{MgSO}_{4}$ and concentrated under reduced pressure at $30{ }^{\circ} \mathrm{C}$ to afford 4-nitrophenyl azide as a slightly yellow solid 
with 98\% yield (32.6 g). 4-nitrophenyl azide was used without further purification. Spectroscopic data for 4-nitrophenyl azide was consistent with previously reported data for this compound. ${ }^{2}$

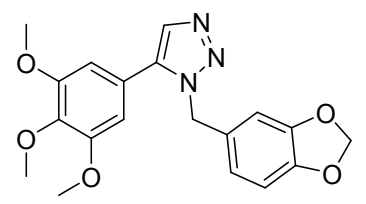

1-(Benzo $[d][1,3]$ dioxol-5-ylmethyl)-5-(3,4,5-trimethoxyphenyl)-1H-1,2,3-triazole $\quad(5) \quad: \quad 3^{\prime}, 4^{\prime}, 5^{\prime}-$ trimethoxyacetophenone ( $200 \mathrm{mg}, 1.0$ equiv., $0.951 \mathrm{mmol})$, piperonylamine (187 mg, 1.3 equiv., 1.237 mmol), 4-nitrophenyl azide (156 mg, 1.0 equiv., $0.951 \mathrm{mmol}), 4 \AA$ molecular sieves $(50 \mathrm{mg})$ and toluene $(0.8 \mathrm{~mL})$, under argon atmosphere. Reaction time is $24 \mathrm{~h}$. The product was purified by flash column chromatography (first with $\mathrm{CH}_{2} \mathrm{Cl}_{2}$ followed by petroleum ether/EtOAc = 3:7) affording $5(334 \mathrm{mg}, 95 \%$ yield) as off white solid. m.p. $126{ }^{\circ} \mathrm{C}$

${ }^{1}$ H NMR $\left(300 \mathrm{MHz}, \mathrm{CDCl}_{3}\right) \delta 7.71(\mathrm{~s}, 1 \mathrm{H}), 6.73(\mathrm{~d}, J=7.9 \mathrm{~Hz}, 1 \mathrm{H}), 6.66(\mathrm{~s}, 1 \mathrm{H}), 6.58(\mathrm{~d}, J=8 \mathrm{~Hz}, 1 \mathrm{H})$, $6.42(\mathrm{~s}, 2 \mathrm{H}), 5.94(\mathrm{~s}, 2 \mathrm{H}), 5.44(\mathrm{~s}, 2 \mathrm{H}), 3.89(\mathrm{~s}, 3 \mathrm{H}), 3.75(\mathrm{~s}, 6 \mathrm{H}) .{ }^{13} \mathbf{C}$ NMR $\left(151 \mathrm{MHz}, \mathrm{CDCl}_{3}\right) \delta 153.5$, $148.1,138.0,133.1,129.5,122.1,120.6,108.3,107.8,106.3,101.3,60.9,56.1,51.6$. HRMS (ESIquadrupole) $m / z:[\mathrm{M}+\mathrm{H}]^{+}$calcd for $\mathrm{C}_{19} \mathrm{H}_{19} \mathrm{~N}_{3} \mathrm{O}_{5} \mathrm{H}, 370.1397$; found, 370.1395 .

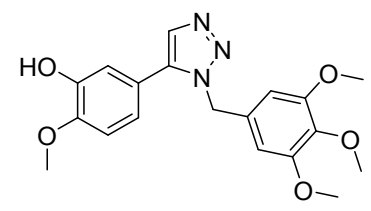

2-Methoxy-5-(1-(3,4,5-trimethoxybenzyl)-1H-1,2,3-triazol-5-yl)-phenol (6): $\quad$ 3'-hydroxy-4'methoxyaceto phenone (200 $\mathrm{mg}, 1.0$ equiv., $1.1204 \mathrm{mmol}$ ), 3,4,5-trimethoxybenzylamine (309 $\mathrm{mg}, 1.3$ equiv., $1.565 \mathrm{mmol})$, 4-nitrophenyl azide (198 mg, 1.0 equiv., $1.1204 \mathrm{mmol}), 4 \AA$ molecular sieves $(50 \mathrm{mg})$ and toluene $(0.8 \mathrm{~mL})$, under argon atmosphere. Reaction time is $24 \mathrm{~h}$. The product was purified by flash column chromatography (first with $\mathrm{CH}_{2} \mathrm{Cl}_{2}$ followed by petroleum ether/EtOAc $\left.=2: 8\right)$ affording $6(326 \mathrm{mg}$, $73 \%$ yield) as off white solid m.p. $183{ }^{\circ} \mathrm{C}$. Spectroscopic data for compound (6) was consistent with previously reported data for this compound. (m.p. literature $\left.194-195^{\circ} \mathrm{C}\right)^{3}$

${ }^{1}$ H NMR $\left(300 \mathrm{MHz}, \mathrm{CDCl}_{3}\right) \delta 7.68(\mathrm{~s}, 1 \mathrm{H}), 6.89(\mathrm{~d}, J=7.7 \mathrm{~Hz}, 2 \mathrm{H}), 6.77(\mathrm{~d}, J=8.3 \mathrm{~Hz}, 1 \mathrm{H}), 6.34(\mathrm{~s}, 2 \mathrm{H})$, $5.82(\mathrm{~s}, 1 \mathrm{H}), 5.45(\mathrm{~s}, 2 \mathrm{H}), 3.94(\mathrm{~s}, 3 \mathrm{H}), 3.81(\mathrm{~s}, 3 \mathrm{H}), 3.76(\mathrm{~s}, 6 \mathrm{H}) .{ }^{13} \mathbf{C} \mathbf{N M R}\left(75 \mathrm{MHz}, \mathrm{CDCl}_{3}\right) \delta 153.49$, 147.6, 146.0, 137.9, 137.8, 133.1, 130.9, 121.1, 120.0, 115.3, 110.9, 104.7, 60.8, 56.1, 52.0. HRMS (ESIquadrupole) $m / z:[\mathrm{M}+\mathrm{H}]^{+}$calcd for $\mathrm{C}_{19} \mathrm{H}_{21} \mathrm{~N}_{3} \mathrm{O}_{5} \mathrm{H}, 372.1553$; found, 372.1544 .

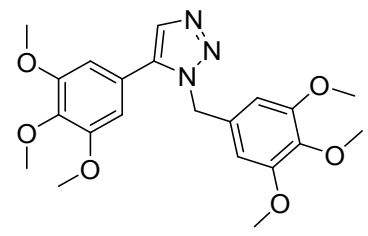

1-(3,4,5-Trimethoxybenzyl)-5-(3,4,5-trimethoxyphenyl)-1 H-1,2,3-triazole (7): 3',4',5'-trimethoxyaceto phenone (200 mg, 1.0 equiv., $0.951 \mathrm{mmol}), 3,4,5$-trimethoxybenzylamine (244 $\mathrm{mg}, 1.3$ equiv., 1.237 
mmol), 4-nitrophenyl azide (156 mg, 1.0 equiv., $0.951 \mathrm{mmol}), 4 \AA$ molecular sieves (50 mg) and toluene $(0.8 \mathrm{~mL})$, under argon atmosphere. Reaction time is $24 \mathrm{~h}$. The product was purified by flash column chromatography (first with $\mathrm{CH}_{2} \mathrm{Cl}_{2}$ followed by petroleum ether/EtOAc = 3:7) affording $7(369 \mathrm{mg}, 93 \%$ yield) as off white solid. m.p. $135{ }^{\circ} \mathrm{C}$

${ }^{1} \mathbf{H}$ NMR $\left(300 \mathrm{MHz}, \mathrm{CDCl}_{3}\right) \delta 7.72(\mathrm{~s}, 1 \mathrm{H}), 6.41(\mathrm{~s}, 2 \mathrm{H}), 6.32(\mathrm{~s}, 2 \mathrm{H}), 5.47(\mathrm{~s}, 2 \mathrm{H}), 3.88(\mathrm{~s}, 3 \mathrm{H}), 3.80(\mathrm{~s}$, $3 \mathrm{H}), 3.75(\mathrm{~d}, J=7.7 \mathrm{~Hz}, 12 \mathrm{H}) .{ }^{13} \mathbf{C}$ NMR $\left(75 \mathrm{MHz}, \mathrm{CDCl}_{3}\right) \delta 153.5,139.1,138.0,137.9,133.2,131.4$, 122.1, 106.5, 104.3, 60.8, 56.1, 52.0. HRMS (ESI-quadrupole) $m / z:[\mathrm{M}+\mathrm{H}]{ }^{+}$calcd for $\mathrm{C}_{21} \mathrm{H}_{25} \mathrm{~N}_{3} \mathrm{O}_{6} \mathrm{H}$, 416.1815; found, 416.1810 .

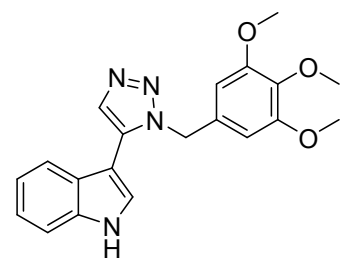

3-(1-(3,4,5-Trimethoxybenzyl)-1H-1,2,3-triazol-5-yl)-1H-indole $(8)$ : 3 -acetylindole (200 mg, 1.0 equiv., $1.256 \mathrm{mmol}$ ), 3,4,5-trimethoxybenzylamine (322 mg, 1.3 equiv., $1.633 \mathrm{mmol})$, 4-nitrophenyl azide (206 mg, 1.0 equiv., $1.256 \mathrm{mmol}), 4 \AA$ molecular sieves $(50 \mathrm{mg})$ and toluene $(0.8 \mathrm{~mL})$, under argon atmosphere. Reaction time is $24 \mathrm{~h}$. The product was purified by flash column chromatography (first with $\mathrm{CH}_{2} \mathrm{Cl}_{2}$ followed by petroleum ether/EtOAc $=1: 9)$ affording $8(326 \mathrm{mg}, 72 \%$ yield $)$ as off white solid. m.p. $178{ }^{\circ} \mathrm{C}$. Spectroscopic data for compound (8) was consistent with previously reported data for this compound. (m.p. literature $\left.180-181^{\circ} \mathrm{C}\right) .{ }^{1}$

${ }^{1}$ H NMR $\left(300 \mathrm{MHz}, \mathrm{CDCl}_{3}\right) \delta 8.91(\mathrm{~s}, 1 \mathrm{H}), 7.89(\mathrm{~s}, 1 \mathrm{H}), 7.53-7.44(\mathrm{~m}, 2 \mathrm{H}), 7.34-7.27(\mathrm{~m}, 1 \mathrm{H}), 7.20$ $(\mathrm{m}, 1 \mathrm{H}), 7.11(\mathrm{~s}, 1 \mathrm{H}), 6.23(\mathrm{~s}, 2 \mathrm{H}), 5.52(\mathrm{~s}, 2 \mathrm{H}), 3.79(\mathrm{~s}, 3 \mathrm{H}), 3.65(\mathrm{~s}, 6 \mathrm{H}) .{ }^{13} \mathbf{C ~ N M R}\left(75 \mathrm{MHz}, \mathrm{CDCl}_{3}\right) \delta$ 153.4, 137.7, 136.0, 133.7, 131.9, 131.4, 126.3, 124.3, 123.3, 121.1, 119.1, 111.7, 104.3, 102.3, 60.8, 56.0, 52.0. HRMS (ESI-quadrupole) $m / z$ : $[\mathrm{M}+\mathrm{H}]^{+}$calcd for $\mathrm{C}_{20} \mathrm{H}_{20} \mathrm{~N}_{4} \mathrm{O}_{3} \mathrm{H}, 365.1608$; found, 365.1607 .

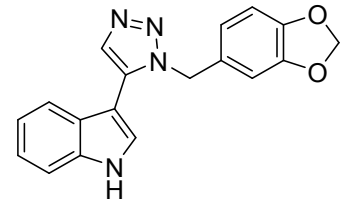

3-(1-(Benzo $[\boldsymbol{d}][\mathbf{1 , 3}]$ dioxol-5-ylmethyl)-1H-1,2,3-triazol-5-yl)-1H-indole (9): 3-acetylindole (200 mg, 1.0 equiv., $1.256 \mathrm{mmol})$, piperonylamine $(247 \mathrm{mg}, 1.3$ equiv., $1.633 \mathrm{mmol})$, 4-nitrophenyl azide (206 $\mathrm{mg}, 1.0$ equiv., $1.256 \mathrm{mmol}), 4 \AA$ molecular sieves $(50 \mathrm{mg})$ and toluene $(0.8 \mathrm{~mL})$, under argon atmosphere. Reaction time is $24 \mathrm{~h}$. The product was purified by flash column chromatography (first with $\mathrm{CH}_{2} \mathrm{Cl}_{2}$ followed by petroleum ether $/$ EtOAc $=2: 8)$ affording $9(361 \mathrm{mg}, 90 \%$ yield $)$ as a brown solid. m.p. $196^{\circ} \mathrm{C}$

${ }^{1} \mathbf{H}$ NMR $\left(300 \mathrm{MHz}, \mathrm{CDCl}_{3}\right) \delta 8.49(\mathrm{~s}, 1 \mathrm{H}), 7.89(\mathrm{~s}, 1 \mathrm{H}), 7.53(\mathrm{~d}, J=7.8 \mathrm{~Hz}, 1 \mathrm{H}), 7.47(\mathrm{~d}, J=7.9 \mathrm{~Hz}, 1 \mathrm{H})$, $7.31(\mathrm{~m}, 1 \mathrm{H}), 7.25-7.18(\mathrm{~m}, 1 \mathrm{H}), 7.10(\mathrm{~s}, 1 \mathrm{H}), 6.70(\mathrm{~d}, J=7.8 \mathrm{~Hz}, 1 \mathrm{H}), 6.63-6.48(\mathrm{~m}, 2 \mathrm{H}), 5.93(\mathrm{~s}, 2 \mathrm{H})$, $5.49(\mathrm{~s}, 2 \mathrm{H}) .{ }^{13} \mathrm{C}$ NMR $\left(151 \mathrm{MHz}, \mathrm{CDCl}_{3}\right) \delta 133.6,126.2,124.0,123.4,121.2,120.5,119.2,111.6,108.4$, 107.6, 101.2, 51.5. HRMS (ESI-quadrupole) $m / z$ : $[\mathrm{M}+\mathrm{H}]{ }^{+}$calcd for $\mathrm{C}_{18} \mathrm{H}_{14} \mathrm{~N}_{4} \mathrm{O}_{2} \mathrm{H}, 319.1189$; found, 319.1190 . 


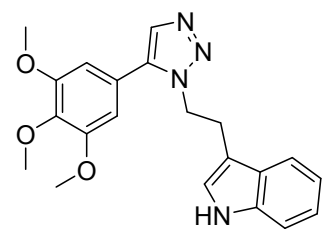

3-(2-(5-(3,4,5-Trimethoxyphenyl)-1H-1,2,3-triazol-1-yl)ethyl)-1 $H$-indole (10): 3',4',5'-trimethoxyaceto phenone (300 mg, 1.0 equiv., $1.427 \mathrm{mmol}$ ), tryptamine (297 mg, 1.3 equiv., $1.855 \mathrm{mmol})$, 4-nitrophenyl azide (234 mg, 1.0 equiv., $1.427 \mathrm{mmol}), 4 \AA$ molecular sieves $(50 \mathrm{mg})$ and toluene $(0.8 \mathrm{~mL})$, under argon atmosphere. Reaction time is $24 \mathrm{~h}$. The product was purified by flash column chromatography (first with $\mathrm{CH}_{2} \mathrm{Cl}_{2}$ followed by petroleum ether/EtOAc $\left.=4: 6\right)$ affording $10(451 \mathrm{mg}, 84 \%$ yield $)$ as a brown solid. m.p. $73{ }^{\circ} \mathrm{C}$

${ }^{1}$ H NMR $\left(300 \mathrm{MHz}, \mathrm{CDCl}_{3}\right) \delta 8.20(\mathrm{~s}, 1 \mathrm{H}), 7.60(\mathrm{~s}, 1 \mathrm{H}), 7.32(\mathrm{~d}, J=8.1 \mathrm{~Hz}, 1 \mathrm{H}), 7.23(\mathrm{~d}, J=8.0 \mathrm{~Hz}, 1 \mathrm{H})$, $7.15(\mathrm{t}, J=7.5 \mathrm{~Hz}, 1 \mathrm{H}), 7.03(\mathrm{t}, J=7.4 \mathrm{~Hz}, 1 \mathrm{H}), 6.74(\mathrm{~s}, 1 \mathrm{H}), 6.17(\mathrm{~s}, 2 \mathrm{H}), 4.61(\mathrm{t}, J=7.2 \mathrm{~Hz}, 2 \mathrm{H}), 3.85$ $(\mathrm{s}, 3 \mathrm{H}), 3.63(\mathrm{~s}, 6 \mathrm{H}), 3.39(\mathrm{t}, J=7.2 \mathrm{~Hz}, 2 \mathrm{H}) .{ }^{13} \mathbf{C} \mathbf{N M R}\left(75 \mathrm{MHz}, \mathrm{CDCl}_{3}\right) \delta 153.4,138.9,138.5,136.1$, $132.8,127.0,122.5,122.3,122.2,119.6,118.1,111.2,111.2,106.2,60.9,56.1,48.7,26.3$. HRMS (ESIquadrupole) $m / z:[\mathrm{M}+\mathrm{H}]^{+}$calcd for $\mathrm{C}_{21} \mathrm{H}_{22} \mathrm{~N}_{4} \mathrm{O}_{3} \mathrm{H}, 379.1764$; found, 379.1764 .

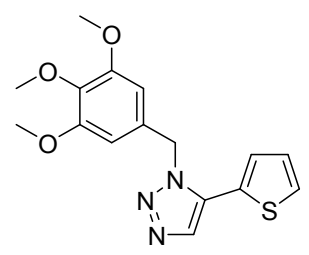

5-(Thiophen-2-yl)-1-(3,4,5-trimethoxybenzyl)-1H-1,2,3-triazole (11) 2-acetylthiophene (200 mg, 1.0 equiv., $1.585 \mathrm{mmol}$ ), 3,4,5-trimethoxybenzylamine (406 mg, 1.3 equiv., $2.061 \mathrm{mmol}$ ), 4-nitrophenyl azide (260 mg, 1.0 equiv., $1.585 \mathrm{mmol}), 4 \AA$ molecular sieves $(50 \mathrm{mg})$ and toluene $(0.8 \mathrm{~mL})$, under argon atmosphere. Reaction time is $24 \mathrm{~h}$. The product was purified by flash column chromatography (first with $\mathrm{CH}_{2} \mathrm{Cl}_{2}$ followed by petroleum ether/EtOAc $\left.=6: 4\right)$ affording $11(511 \mathrm{mg}, 97 \%$ yield $)$ as slightly yellow semi solid.

${ }^{1} \mathbf{H}$ NMR $\left(300 \mathrm{MHz}, \mathrm{CDCl}_{3}\right) \delta 7.80(\mathrm{~s}, 1 \mathrm{H}), 7.48(\mathrm{~d}, J=5.1 \mathrm{~Hz}, 1 \mathrm{H}), 7.17-7.10(\mathrm{~m}, 1 \mathrm{H}), 7.08(\mathrm{~d}, J=3.5$ $\mathrm{Hz}, 1 \mathrm{H}), 6.32(\mathrm{~s}, 2 \mathrm{H}), 5.57(\mathrm{~s}, 2 \mathrm{H}), 3.81(\mathrm{~s}, 3 \mathrm{H}), 3.76(\mathrm{~s}, 6 \mathrm{H}) .{ }^{13} \mathbf{C}$ NMR $\left(75 \mathrm{MHz}, \mathrm{CDCl}_{3}\right) \delta 153.5,138.0$, 134.0, 131.6, 130.6, 128.7, 128.2, 127.9, 126.7, 104.4, 60.8, 56.1, 52.2. HRMS (ESI-quadrupole) $\mathrm{m} / z$ : [M $+\mathrm{H}]^{+}$calcd for $\mathrm{C}_{16} \mathrm{H}_{17} \mathrm{~N}_{3} \mathrm{O}_{3} \mathrm{~S}_{1} \mathrm{H}, 332.1063$; found, 332.1057 .

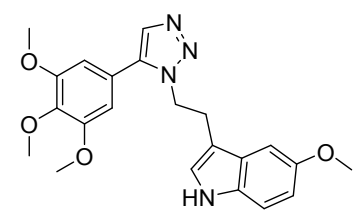

5-Methoxy-3-(2-(5-(3,4,5-trimethoxyphenyl)-1H-1,2,3-triazol-1-yl)ethyl)-1H-indole $\quad(12): \quad 3^{\prime}, 4^{\prime}, 5^{\prime}-$ trimethoxyaceto phenone ( $200 \mathrm{mg}, 1.0$ equiv., $0.951 \mathrm{mmol}), 5$-methoxytryptamine ( $235 \mathrm{mg}$, 1.3 equiv., $1.237 \mathrm{mmol}), 4$-nitrophenyl azide (234 mg, 1.0 equiv., $0.951 \mathrm{mmol}), 4 \AA$ molecular sieves $(50 \mathrm{mg})$ and toluene $(0.8 \mathrm{~mL})$, under argon atmosphere. Reaction time is $24 \mathrm{~h}$. The product was purified by flash column 
chromatography (first with $\mathrm{CH}_{2} \mathrm{Cl}_{2}$ followed by petroleum ether/EtOAc = 3:7) affording $12(291 \mathrm{mg}, 75 \%$ yield) as off white solid. m.p. $152{ }^{\circ} \mathrm{C}$.

${ }^{1}$ H NMR $(300 \mathrm{MHz}, \mathrm{CDCl} 3) \delta 7.87(\mathrm{~s}, 1 \mathrm{H}), 7.58(\mathrm{~s}, 1 \mathrm{H}), 7.18(\mathrm{~d}, \mathrm{~J}=8.8 \mathrm{~Hz}, 1 \mathrm{H}), 6.81(\mathrm{dd}, \mathrm{J}=8.7,2.2$ $\mathrm{Hz}, 1 \mathrm{H}), 6.73(\mathrm{~d}, \mathrm{~J}=2.1 \mathrm{~Hz}, 1 \mathrm{H}), 6.60(\mathrm{~d}, \mathrm{~J}=2.1 \mathrm{~Hz}, 1 \mathrm{H}), 6.12(\mathrm{~s}, 2 \mathrm{H}), 4.59(\mathrm{t}, \mathrm{J}=6.8 \mathrm{~Hz}, 2 \mathrm{H}), 3.83(\mathrm{~s}$, $3 \mathrm{H}), 3.77(\mathrm{~s}, 3 \mathrm{H}), 3.64(\mathrm{~s}, 6 \mathrm{H}), 3.38(\mathrm{t}, \mathrm{J}=6.6 \mathrm{~Hz}, 2 \mathrm{H}) .{ }^{13} \mathbf{C}$ NMR $(101 \mathrm{MHz}, \mathrm{CDCl} 3) \delta 154.2,153.3,138.7$, 138.6, 132.8, 131.1, 127.5, 123.2, 122.2, 112.6, 111.8, 111.3, 106.0, 99.6, 60.8, 56.0, 55.7, 48.8, 26.3. HRMS (ESI-quadrupole) $m / z:[\mathrm{M}+\mathrm{H}]^{+}$calcd for $\mathrm{C}_{22} \mathrm{H}_{24} \mathrm{~N}_{4} \mathrm{O}_{4} \mathrm{H}, 409.1870$; found, 409.1866 .

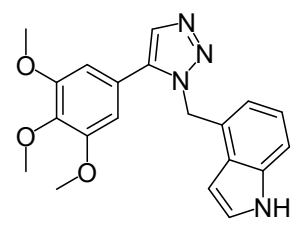

4-((5-(3,4,5-Trimethoxyphenyl)-1 $H$-1,2,3-triazol-1-yl)methyl)- $1 H$-indole (13): 3', $4^{\prime}, 5^{\prime}$-trimethoxyaceto phenone (200 mg, 1.0 equiv., $0.951 \mathrm{mmol}), 5$-methoxytryptamine (181 mg, 1.3 equiv., $1.237 \mathrm{mmol}), 4-$ nitrophenyl azide (156 mg, 1.0 equiv., $0.951 \mathrm{mmol}), 4 \AA$ molecular sieves $(50 \mathrm{mg})$ and toluene $(0.8 \mathrm{~mL})$, under argon atmosphere. Reaction time is $24 \mathrm{~h}$. The product was purified by flash column chromatography (first with $\mathrm{CH}_{2} \mathrm{Cl}_{2}$ followed by petroleum ether/EtOAc $=5: 5$ ) affording 13 (299 $\mathrm{mg}, 86 \%$ yield) as orange solid. m.p. $53{ }^{\circ} \mathrm{C}$.

${ }^{1} \mathbf{H}$ NMR (300 MHz, CDCl3) $\delta 8.37(\mathrm{~s}, 1 \mathrm{H}), 7.75(\mathrm{~s}, 1 \mathrm{H}), 7.33(\mathrm{~d}, \mathrm{~J}=8.2 \mathrm{~Hz}, 1 \mathrm{H}), 7.24-7.19(\mathrm{~m}, 1 \mathrm{H})$, $7.10(\mathrm{t}, \mathrm{J}=7.7 \mathrm{~Hz}, 1 \mathrm{H}), 6.70-6.63(\mathrm{~m}, 1 \mathrm{H}), 6.45-6.40(\mathrm{~m}, 1 \mathrm{H}), 6.33(\mathrm{~s}, 2 \mathrm{H}), 5.85(\mathrm{~s}, 2 \mathrm{H}), 3.82(\mathrm{~s}, 3 \mathrm{H})$, 3.47 (s, 6H). ${ }^{13}$ C NMR (101 MHz, CDCl3) $\delta 153.3,138.6,138.3,135.8,133.0,127.7,125.3,124.7,122.2$, 122.1, 118.2, 111.0, 105.9, 100.2, 60.8, 55.7, 50.4. HRMS (ESI-quadrupole) $m / z:[\mathrm{M}+\mathrm{H}]{ }^{+}$calcd for $\mathrm{C}_{20} \mathrm{H}_{20} \mathrm{~N}_{4} \mathrm{O}_{3} \mathrm{H}, 365.1608$; found, 365.1599.

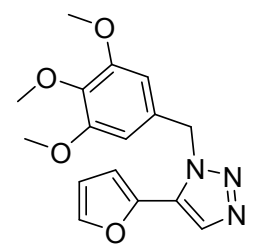

5-(Furan-2-yl)-1-(3,4,5-trimethoxybenzyl)-1H-1,2,3-triazole (14): 2-acetylfuran (200 mg, 1.0 equiv., $1.816 \mathrm{mmol}$ ), 3,4,5-trimethoxybenzylamine (466 mg, 1.3 equiv., $2.361 \mathrm{mmol}$ ), 4-nitrophenyl azide (298 mg, 1.0 equiv., $1.816 \mathrm{mmol}), 4 \AA$ molecular sieves $(50 \mathrm{mg})$ and toluene $(0.8 \mathrm{~mL})$, under argon atmosphere. Reaction time is $24 \mathrm{~h}$. The product was purified by flash column chromatography (first with $\mathrm{CH}_{2} \mathrm{Cl}_{2}$ followed by petroleum ether/EtOAc $=6: 4)$ affording $14(467 \mathrm{mg}, 82 \%$ yield $)$ as orange solid. m.p. $62{ }^{\circ} \mathrm{C}$.

${ }^{1}$ H NMR $(300 \mathrm{MHz}, \mathrm{CDCl} 3) \delta 7.86(\mathrm{~s}, 1 \mathrm{H}), 7.57(\mathrm{dd}, \mathrm{J}=1.8,0.7 \mathrm{~Hz}, 1 \mathrm{H}), 6.56(\mathrm{dd}, \mathrm{J}=3.4,0.7 \mathrm{~Hz}, 1 \mathrm{H})$, $6.51(\mathrm{dd}, \mathrm{J}=3.4,1.8 \mathrm{~Hz}, 1 \mathrm{H}), 6.39(\mathrm{~s}, 2 \mathrm{H}), 5.69(\mathrm{~s}, 2 \mathrm{H}), 3.80(\mathrm{~s}, 3 \mathrm{H}), 3.76(\mathrm{~s}, 6 \mathrm{H}) .{ }^{13} \mathrm{C}$ NMR $(101 \mathrm{MHz}$, $\mathrm{CDCl} 3) \delta 153.4,143.6,141.3,137.8,132.4,130.6,128.9,111.9,110.6,104.3,60.7,56.0,52.9$. HRMS (ESI-quadrupole) $\mathrm{m} / \mathrm{z}:[\mathrm{M}+\mathrm{H}]^{+}$calcd for $\mathrm{C}_{16} \mathrm{H}_{17} \mathrm{~N}_{3} \mathrm{O}_{4} \mathrm{H}, 316.1291$; found, 316.1283 


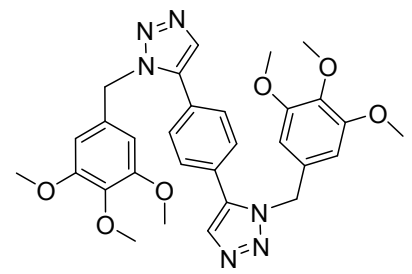

1,4-bis(1-(3,4,5-trimethoxybenzyl)-1H-1,2,3-triazol-5-yl)benzene (15): 1,4-diacetylbenzene (200 mg, 1.0 equiv., $1.233 \mathrm{mmol}), 3,4,5$-trimethoxybenzylamine (632 $\mathrm{mg}, 2.6$ equiv., $3.21 \mathrm{mmol})$, 4-nitrophenyl azide (405 mg, 2.0 equiv., $2.466 \mathrm{mmol}), 4 \AA$ molecular sieves $(50 \mathrm{mg})$ and toluene $(0.8 \mathrm{~mL})$, under argon atmosphere. Reaction time is $24 \mathrm{~h}$. The product was purified by flash column chromatography (first with $\mathrm{CH}_{2} \mathrm{Cl}_{2}$ followed by petroleum ether/EtOAc $\left.=0: 10\right)$ affording $15(555 \mathrm{mg}, 79 \%$ yield $)$ as slightly pink solid. m.p. $210{ }^{\circ} \mathrm{C}$.

${ }^{1}$ H NMR (300 MHz, CDCl3) $\delta 7.77$ (s, 2H), 7.35 (s, 4H), $6.28(\mathrm{~s}, 4 \mathrm{H}), 5.50$ (s, 4H), $3.81(\mathrm{~s}, 6 \mathrm{H}), 3.72(\mathrm{~s}$, 12H). ${ }^{13}$ C NMR (101 MHz, CDCl3) $\delta 153.6,137.9,136.9,133.5,130.6,129.4,128.2,104.3,60.8,56.1$, 52.2. HRMS (ESI-quadrupole) $m / z$ : $[\mathrm{M}+\mathrm{H}]^{+}$calcd for $\mathrm{C}_{30} \mathrm{H}_{32} \mathrm{~N}_{6} \mathrm{O}_{6} \mathrm{H}, 573.2455$; found, 573.2431.

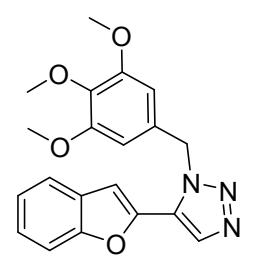

5-(Benzofuran-2-yl)-1-(3,4,5-trimethoxybenzyl)-1H-1,2,3-triazole (16): 2-acetylbenzofuran (200 mg, 1.0 equiv., $1.249 \mathrm{mmol}), 3,4,5$-trimethoxybenzylamine (205 mg, 1.3 equiv., $1.249 \mathrm{mmol}$ ), 4-nitrophenyl azide (320 mg, 1.0 equiv., $1.623 .749 \mathrm{mmol}), 4 \AA$ molecular sieves $(50 \mathrm{mg})$ and toluene $(0.8 \mathrm{~mL})$, under argon atmosphere. Reaction time is $24 \mathrm{~h}$. The product was purified by flash column chromatography (first with $\mathrm{CH}_{2} \mathrm{Cl}_{2}$ followed by petroleum ether/EtOAc $\left.=6: 4\right)$ affording $16(343 \mathrm{mg}, 75 \%$ yield $)$ as slightly yellow solid. m.p. $71{ }^{\circ} \mathrm{C}$.

${ }^{1}$ H NMR (300 MHz, CDCl3) $\delta 8.02(\mathrm{~s}, 1 \mathrm{H}), 7.57(\mathrm{dd}, \mathrm{J}=13.1,8.3 \mathrm{~Hz}, 2 \mathrm{H}), 7.37$ (t, J = 7.7 Hz, 1H), 7.28 $(\mathrm{dd}, \mathrm{J}=9.1,5.8 \mathrm{~Hz}, 1 \mathrm{H}), 6.91(\mathrm{~s}, 1 \mathrm{H}), 6.43(\mathrm{~s}, 2 \mathrm{H}), 5.80(\mathrm{~s}, 2 \mathrm{H}), 3.77(\mathrm{~s}, 3 \mathrm{H}), 3.66(\mathrm{~s}, 6 \mathrm{H}) .{ }^{13} \mathbf{C} \mathbf{N M R}(101$ $\mathrm{MHz}, \mathrm{CDCl} 3) \delta 154.8,153.4,142.9,137.8,133.7,130.3,128.6,127.7,125.8,123.8,121.6,111.2,106.9$, 104.5, 60.7, 55.9, 53.4. HRMS (ESI-quadrupole) $\mathrm{m} / z$ : $[\mathrm{M}+\mathrm{H}]^{+}$calcd for $\mathrm{C}_{20} \mathrm{H}_{19} \mathrm{~N}_{3} \mathrm{O}_{4} \mathrm{H}, 366.1448$; found, 366.1436 .

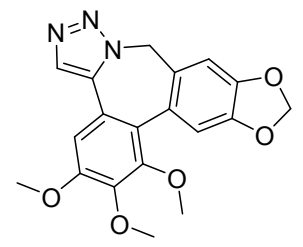

1,2,3-Trimethoxy-9H-[1,3]dioxolo[4',5':4,5] benzo[1,2-e $]$ benzo $[c][1,2,3]$ triazolo[1,5-a] azepine $\quad(5 a)$ : To a stirred solution of compound $5(100 \mathrm{mg}, 1.0$ equiv., $0,271 \mathrm{mmol})$ in dry dichloromethane $(2.0 \mathrm{~mL})$ was added a solution of phenyliodine bis(trifluoroacetate) PIFA (128 mg, 1.1 equiv., $0.298 \mathrm{mmol})$ in dry dichloromethane $(2.0 \mathrm{~mL})$ and $\mathrm{BF}_{3} \cdot \mathrm{Et}_{2} \mathrm{O}(100 \mu \mathrm{L}, 3.0$ equiv., 0.812$)$ at $-40{ }^{\circ} \mathrm{C}$ under argon atmosphere and 
the mixture stirred for $3 \mathrm{~h}$. After confirmation that the starting material is consumed via TLC the reaction was quenched with $\mathrm{NaHCO}_{3}$ solution and extracted 3x with DCM, dried over $\mathrm{MgSO}_{4}$ and evaporated under reduced pressure. The crude was purified via column chromatography (silica gel) were as eluent was used petroleum ether/EtOAc $=6: 4$. Affording 5a (74 mg, 74\% yield) as off white solid. m.p. $180{ }^{\circ} \mathrm{C}$.

${ }^{1}$ H NMR $\left(300 \mathrm{MHz}, \mathrm{CDCl}_{3}\right) \delta 7.81(\mathrm{~s}, 1 \mathrm{H}), 7.13(\mathrm{~s}, 1 \mathrm{H}), 6.94(\mathrm{~s}, 1 \mathrm{H}), 6.84(\mathrm{~s}, 1 \mathrm{H}), 6.04(\mathrm{~s}, 1 \mathrm{H}), 5.96(\mathrm{~s}$, $1 \mathrm{H}), 5.53(\mathrm{~d}, J=13.9 \mathrm{~Hz}, 1 \mathrm{H}), 4.89(\mathrm{~d}, J=13.9 \mathrm{~Hz}, 1 \mathrm{H}), 3.98(\mathrm{~d}, J=11.7 \mathrm{~Hz}, 6 \mathrm{H}), 3.68(\mathrm{~s}, 3 \mathrm{H}) .{ }^{13} \mathbf{C} \mathbf{N M R}$ $\left(151 \mathrm{MHz}, \mathrm{CDCl}_{3}\right) \delta 153.1,152.2,147.5,147.4,143.5,135.9,131.3,129.4,127.5,124.1,122.0,112.2$, 108.2, 107.7, 101.6, 61.2, 61.1, 56.2, 51.5. HRMS (ESI-quadrupole) $\mathrm{m} / z$ : $[\mathrm{M}+\mathrm{H}]^{+}$calcd for $\mathrm{C}_{19} \mathrm{H}_{17} \mathrm{~N}_{3} \mathrm{O}_{5} \mathrm{H}$, 368.1240; found, 368.1241.

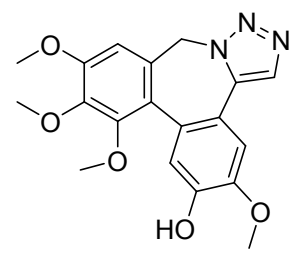

3,11,12,13-Tetramethoxy-9H-dibenzo $[c, e][1,2,3]$ triazolo[1,5-a] azepin-2-ol (6a): To a stirred solution of compound 6 (100 mg, 1.0 equiv., $0,269 \mathrm{mmol})$ in dry dichloromethane $(2.0 \mathrm{~mL})$ was added a solution of phenyliodine bis(trifluoroacetate) PIFA $(127 \mathrm{mg}, 1.1$ equiv., $0.296 \mathrm{mmol})$ in dry dichloromethane $(2.0 \mathrm{~mL})$ and $\mathrm{BF}_{3} \cdot \mathrm{Et}_{2} \mathrm{O}(100 \mu \mathrm{L}, 3.0$ equiv., 0.808$)$ at $-40{ }^{\circ} \mathrm{C}$ under argon atmosphere and the mixture stirred for $3 \mathrm{~h}$. After confirmation that the starting material is consumed via TLC the reaction was quenched with $\mathrm{NaHCO}_{3}$ solution and extracted 3x with DCM, dried over $\mathrm{MgSO}_{4}$ and evaporated under reduced pressure. The crude was purified via reparative TLC were as eluent was used DCM/Acetone = 9:1. Affording 6a (23 mg, 23\% yield) as off white solid. m.p. $226^{\circ} \mathrm{C}$.

${ }^{1} \mathrm{H}$ NMR $\left(600 \mathrm{MHz}, \mathrm{C}_{2} \mathrm{D}_{2} \mathrm{Cl}_{4}\right) \delta 7.78(\mathrm{~s}, 1 \mathrm{H}), 7.37(\mathrm{~s}, 1 \mathrm{H}), 7.12(\mathrm{~s}, 1 \mathrm{H}), 6.78(\mathrm{~s}, 1 \mathrm{H}), 5.52(\mathrm{~d}, J=13.9 \mathrm{~Hz}$, $1 \mathrm{H}), 4.90(\mathrm{~d}, J=13.9 \mathrm{~Hz}, 1 \mathrm{H}), 3.97(\mathrm{~s}, 3 \mathrm{H}), 3.90(\mathrm{~s}, 3 \mathrm{H}), 3.86(\mathrm{~s}, 3 \mathrm{H}), 3.48(\mathrm{~s}, 3 \mathrm{H}) .{ }^{13} \mathrm{C}$ NMR $(151 \mathrm{MHz}$, $\left.\mathrm{C}_{2} \mathrm{D}_{2} \mathrm{Cl}_{4}\right) \delta 146.4,145.1,142.7,130.8,125.2,123.9,114.5,114.3,107.4,61.1,60.9,56.2,56.0,52.0$. HRMS (ESI-quadrupole) $m / z:[\mathrm{M}+\mathrm{H}]^{+}$calcd for $\mathrm{C}_{19} \mathrm{H}_{19} \mathrm{~N}_{3} \mathrm{O}_{5} \mathrm{H}, 370.1397$; found, 370.1397.

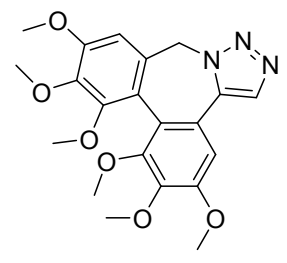

1,2,3,11,12,13-Hexamethoxy-9H-dibenzo $[c, e][1,2,3]$ triazolo[1,5-a]azepine (7a): To a stirred solution of compound 7 (100 mg, 1.0 equiv., $0,241 \mathrm{mmol})$ in dry dichloromethane $(2.0 \mathrm{~mL})$ was added a solution of phenyliodine bis(trifluoroacetate) PIFA $(114 \mathrm{mg}, 1.1$ equiv., $0.265 \mathrm{mmol})$ in dry dichloromethane $(2.0 \mathrm{~mL})$ and $\mathrm{BF}_{3} \cdot \mathrm{Et}_{2} \mathrm{O}(90 \mu \mathrm{L}, 3.0$ equiv., 0.722$)$ at $-40{ }^{\circ} \mathrm{C}$ under argon atmosphere and the mixture stirred for $3 \mathrm{~h}$. After confirmation that the starting material is consumed via TLC the reaction was quenched with $\mathrm{NaHCO}_{3}$ solution and extracted 3x with DCM, dried over $\mathrm{MgSO}_{4}$ and evaporated under reduced pressure. The crude was purified via column chromatography (silica gel) were as eluent was used petroleum ether/EtOAc $=2: 8$. Affording $7 \mathbf{a}\left(55 \mathrm{mg}, 55 \%\right.$ yield) as off white solid. m.p. $82{ }^{\circ} \mathrm{C}$. 
${ }^{1}$ H NMR $\left(300 \mathrm{MHz}, \mathrm{CDCl}_{3}\right) \delta 7.83(\mathrm{~s}, 1 \mathrm{H}), 6.83(\mathrm{~s}, 1 \mathrm{H}), 6.77(\mathrm{~s}, 1 \mathrm{H}), 5.54(\mathrm{~d}, J=13.8 \mathrm{~Hz}, 1 \mathrm{H}), 4.93(\mathrm{~d}, J$ $=13.8 \mathrm{~Hz}, 1 \mathrm{H}), 3.96(\mathrm{~s}, 6 \mathrm{H}), 3.90(\mathrm{~s}, 3 \mathrm{H}), 3.84(\mathrm{~s}, 3 \mathrm{H}), 3.75(\mathrm{~s}, 3 \mathrm{H}), 3.61(\mathrm{~s}, 3 \mathrm{H}) .{ }^{13} \mathbf{C}$ NMR $(75 \mathrm{MHz}$, $\left.\mathrm{CDCl}_{3}\right) \delta 153.5,153.5,153.1,152.9,142.5,142.2,135.9,132.3,131.0,122.3,120.5,120.4,107.1,106.6$, 61.3, 61.1, 61.0, 60.9, 56.2, 56.0. HRMS (ESI-quadrupole) $m / z$ : $[\mathrm{M}+\mathrm{H}]{ }^{+}$calcd for $\mathrm{C}_{21} \mathrm{H}_{23} \mathrm{~N}_{3} \mathrm{O}_{6} \mathrm{H}$, 414.1659; found, 414.1649.

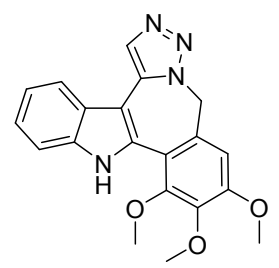

11,12,13-Trimethoxy-9,14-dihydrobenzo[5,6][1,2,3]triazolo $\left[1^{\prime}, 5^{\prime}: 1,2\right]$ azepino $[4,3-b]$ indole $(8 a)$ : To a stirred solution of compound 8 (100 $\mathrm{mg}, 1.0$ equiv., $0,274 \mathrm{mmol})$ in dry dichloromethane $(2.0 \mathrm{~mL})$ was added a solution of phenyliodine bis(trifluoroacetate) PIFA (130 mg, 1.1 equiv., $0.302 \mathrm{mmol})$ in dry dichloromethane $(2.0 \mathrm{~mL})$ and $\mathrm{BF}_{3} \cdot \mathrm{Et}_{2} \mathrm{O}(102 \mu \mathrm{L}, 3.0$ equiv., 0.823$)$ at $-40{ }^{\circ} \mathrm{C}$ under argon atmosphere and the mixture stirred for $3 \mathrm{~h}$. After confirmation that the starting material is consumed via TLC the reaction was quenched with $\mathrm{NaHCO}_{3}$ solution and extracted 3x with DCM, dried over $\mathrm{MgSO}_{4}$ and evaporated under reduced pressure. The crude was purified via column chromatography (silica gel) were as eluent was used petroleum ether/EtOAc $=1: 9$. Affording $8 \mathbf{a}\left(93.7 \mathrm{mg}, 94 \%\right.$ yield) as off white solid. m.p. $187^{\circ} \mathrm{C}$.

1 mmol scale synthesis of (8a): To a stirred solution of compound 8 (400 mg, 1.0 equiv., 1,098 mmol) in dry dichloromethane $(8.0 \mathrm{~mL})$ was added a solution of phenyliodine bis(trifluoroacetate) PIFA (519 mg, 1.1 equiv., $1.207 \mathrm{mmol})$ in dry dichloromethane $(8 \mathrm{~mL})$ and $\mathrm{BF}_{3} \cdot \mathrm{Et}_{2} \mathrm{O}(406 \mu \mathrm{L}, 3.0$ equiv., 3.29$)$ at $-40{ }^{\circ} \mathrm{C}$ under argon atmosphere and the mixture stirred for $3 \mathrm{~h}$. After confirmation that the starting material is consumed via TLC the reaction was quenched with $\mathrm{NaHCO}_{3}$ solution and extracted 3x with DCM, dried over $\mathrm{MgSO}_{4}$ and evaporated under reduced pressure. The crude was purified via column chromatography (silica gel) were as eluent was used petroleum ether/EtOAc $=1$ :9. Affording $\mathbf{8 a}(310 \mathrm{mg}, 78 \%$ yield) as off white solid.

${ }^{1} \mathbf{H}$ NMR $\left(300 \mathrm{MHz}, \mathrm{CDCl}_{3}\right) \delta 8.02(\mathrm{~s}, 1 \mathrm{H}), 7.76(\mathrm{~d}, J=7.7 \mathrm{~Hz}, 1 \mathrm{H}), 7.40(\mathrm{t}, J=7.6 \mathrm{~Hz}, 1 \mathrm{H}), 7.17(\mathrm{t}, J=$ $7.5 \mathrm{~Hz}, 1 \mathrm{H}), 7.09(\mathrm{~s}, 1 \mathrm{H}), 6.96(\mathrm{~d}, J=7.4 \mathrm{~Hz}, 1 \mathrm{H}), 6.71(\mathrm{~s}, 1 \mathrm{H}), 5.78(\mathrm{~s}, 2 \mathrm{H}), 3.94(\mathrm{~s}, 3 \mathrm{H}), 3.76(\mathrm{~s}, 3 \mathrm{H})$, $3.11(\mathrm{~s}, 3 \mathrm{H}) .{ }^{13} \mathrm{C}$ NMR $\left(151 \mathrm{MHz}, \mathrm{CDCl}_{3}\right) \delta 172.0,155.0,154.4,152.1,143.7,141.9,130.6,129.2,128.9$, 127.2, 125.3, 121.7, 114.7, 104.8, 60.6, 60.2, 57.3, 56.1, 47.7. HRMS (ESI-quadrupole) $\mathrm{m} / \mathrm{z}:[\mathrm{M}+\mathrm{H}]^{+}$ calcd for $\mathrm{C}_{20} \mathrm{H}_{18} \mathrm{~N}_{4} \mathrm{O}_{3} \mathrm{H}, 363.1451$; found, 363.1452 .

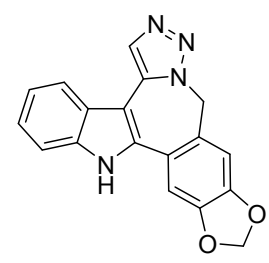

9,15-Dihydro-[1,3]dioxolo [4', $\left.5^{\prime \prime}: 4^{\prime}, 5^{\prime}\right]$ benzo $\left[1^{\prime}, 2^{\prime}: 5,6\right][1,2,3]$ triazolo $\left[1^{\prime}, 5^{\prime}: 1,2\right]$ azepino $[4,3-b]$ indole

(9a) : To a stirred solution of compound 9 (100 mg, 1.0 equiv., $0,314 \mathrm{mmol})$ in dry dichloromethane $(2.0$ $\mathrm{mL}$ ) was added a solution of phenyliodine bis(trifluoroacetate) PIFA (149 mg, 1.1 equiv., $0.346 \mathrm{mmol}$ ) in dry dichloromethane $(2.0 \mathrm{~mL})$ and $\mathrm{BF}_{3} \cdot \mathrm{Et}_{2} \mathrm{O}(116 \mu \mathrm{L}, 3.0$ equiv., 0.942$)$ at $-40{ }^{\circ} \mathrm{C}$ under argon atmosphere 
and the mixture stirred for $3 \mathrm{~h}$. After confirmation that the starting material is consumed via TLC the reaction was quenched with $\mathrm{NaHCO}_{3}$ solution and extracted $3 \mathrm{x}$ with $\mathrm{DCM}$, dried over $\mathrm{MgSO}_{4}$ and evaporated under reduced pressure. The crude was purified via column chromatography (silica gel) were as eluent was used petroleum ether/EtOAc $=1: 9$. Affording $9 \mathrm{a}(61.7 \mathrm{mg}, 62 \%$ yield $)$ as off white solid. m.p. $208^{\circ} \mathrm{C}$.

${ }^{1} \mathbf{H}$ NMR $\left(300 \mathrm{MHz}, \mathrm{CDCl}_{3}\right) \delta 7.90(\mathrm{~s}, 1 \mathrm{H}), 7.79(\mathrm{~d}, J=7.7 \mathrm{~Hz}, 1 \mathrm{H}), 7.51(\mathrm{t}, J=7.6 \mathrm{~Hz}, 1 \mathrm{H}), 7.31(\mathrm{t}, J=$ $7.5 \mathrm{~Hz}, 1 \mathrm{H}), 7.12(\mathrm{~d}, J=9.3 \mathrm{~Hz}, 2 \mathrm{H}), 6.87(\mathrm{~s}, 1 \mathrm{H}), 5.99-5.95(\mathrm{~m}, 3 \mathrm{H}), 5.75(\mathrm{~m}, 2 \mathrm{H}) .{ }^{13} \mathbf{C} \mathbf{N M R}(151 \mathrm{MHz}$, $\left.\mathrm{CDCl}_{3}\right) \delta 171.3,155.4,148.7,148.5,141.9,130.5,129.8,129.6,128.1,123.3,123.2,122.1,121.2,106.6$, 105.6, 101.9, 59.9, 48.2. HRMS (ESI-quadrupole) $\mathrm{m} / \mathrm{z}:[\mathrm{M}+\mathrm{H}]^{+}$calcd for $\mathrm{C}_{18} \mathrm{H}_{12} \mathrm{~N}_{4} \mathrm{O}_{2} \mathrm{H}, 317.1032$; found, 317.1031 .

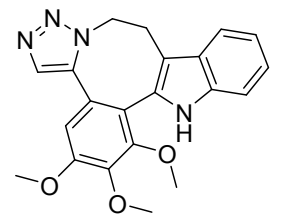

1,2,3-Trimethoxy-10,15-dihydro-9H-benzo[6,7][1,2,3]triazolo[ $\left[1^{\prime}, 5^{\prime}: 1,8\right]$ azocino[5,4-b]indole (10a) : To a stirred solution of compound $\mathbf{1 0}(100 \mathrm{mg}, 1.0$ equiv., $0,264 \mathrm{mmol})$ in dry dichloromethane $(2.0 \mathrm{~mL})$ was added a solution of phenyliodine bis(trifluoroacetate) PIFA (125 mg, 1.1 equiv., $0.291 \mathrm{mmol})$ in dry dichloromethane $(2.0 \mathrm{~mL})$ and $\mathrm{BF}_{3} \cdot \mathrm{Et}_{2} \mathrm{O}(98 \mu \mathrm{L}, 3.0$ equiv., 0.793$)$ at $-40{ }^{\circ} \mathrm{C}$ under argon atmosphere and the mixture stirred for $3 \mathrm{~h}$. After confirmation that the starting material is consumed via TLC the reaction was quenched with $\mathrm{NaHCO}_{3}$ solution and extracted $3 \mathrm{x}$ with $\mathrm{DCM}$, dried over $\mathrm{MgSO}_{4}$ and evaporated under reduced pressure. The crude was purified via column chromatography (silica gel) were as eluent was used petroleum ether/EtOAc = 3:7. Affording 10a (30 mg, 30\% yield) as off white solid. m.p. $184{ }^{\circ} \mathrm{C}$.

${ }^{1} \mathbf{H}$ NMR $\left(300 \mathrm{MHz}, \mathrm{CDCl}_{3}\right) \delta 8.22(\mathrm{~s}, 1 \mathrm{H}), 8.02(\mathrm{~s}, 1 \mathrm{H}), 7.72(\mathrm{~d}, J=7.6 \mathrm{~Hz}, 1 \mathrm{H}), 7.39(\mathrm{t}, J=7.5 \mathrm{~Hz}, 1 \mathrm{H})$, 7.19 (t, $J=7.4 \mathrm{~Hz}, 1 \mathrm{H}), 7.06(\mathrm{~s}, 1 \mathrm{H}), 7.01(\mathrm{~d}, J=7.3 \mathrm{~Hz}, 1 \mathrm{H}), 4.76(\mathrm{~m}, 2 \mathrm{H}), 3.94(\mathrm{~s}, 3 \mathrm{H}), 3.77(\mathrm{~s}, 3 \mathrm{H}), 3.00$ (s, 3H), $2.42-2.32(\mathrm{~m}, 1 \mathrm{H}), 1.79(\mathrm{~m}, 1 \mathrm{H}) .{ }^{13} \mathbf{C} \mathbf{N M R}\left(151 \mathrm{MHz}, \mathrm{CDCl}_{3}\right) \delta 177.1,153.3,153.1,144.6$, 143.0, 136.8, 134.2, 128.4, 126.4, 121.9, 121.8, 121.4, 108.7, 63.0, 60.6, 60.3, 56.1, 46.8, 34.7. HRMS (ESI-quadrupole) $m / z:[\mathrm{M}+\mathrm{H}]^{+}$calcd for $\mathrm{C}_{21} \mathrm{H}_{20} \mathrm{~N}_{4} \mathrm{O}_{3} \mathrm{H}, 377.1608$; found, 377.1602 .

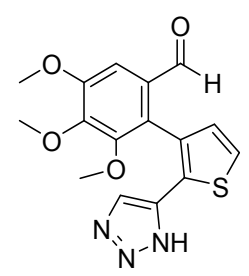

2-(2-(1H-1,2,3-triazol-5-yl)thiophen-3-yl)-3,4,5-trimethoxybenzaldehyde (11ab) : To a stirred solution of compound $11(100 \mathrm{mg}, 1.0$ equiv., $0.302 \mathrm{mmol})$ in dry dichloromethane $(2.0 \mathrm{~mL})$ was added a solution of phenyliodine bis(trifluoroacetate) PIFA (143 mg, 1.1 equiv., $0.332 \mathrm{mmol})$ in dry dichloromethane $(2.0$ $\mathrm{mL})$ and $\mathrm{BF}_{3} \cdot \mathrm{Et}_{2} \mathrm{O}(112 \mu \mathrm{L}, 3.0$ equiv., 0.905$)$ at $-40{ }^{\circ} \mathrm{C}$ under argon atmosphere and the mixture stirred for $3 \mathrm{~h}$. After confirmation that the starting material is consumed via TLC the reaction was quenched with $\mathrm{NaHCO}_{3}$ solution and extracted 3x with DCM, dried over $\mathrm{MgSO}_{4}$ and evaporated under reduced pressure. The crude was purified via column chromatography (silica gel) were as eluent was used Petroleum ether/EtOAc $=4: 6$. Affording $11 \mathbf{a b}(30 \mathrm{mg}, 30 \%$ yield) as slightly yellow semi solid. 
${ }^{1} \mathbf{H}$ NMR $\left(300 \mathrm{MHz}, \mathrm{CDCl}_{3}\right) \delta 9.88(\mathrm{~s}, 1 \mathrm{H}), 7.88(\mathrm{~s}, 1 \mathrm{H}), 7.43(\mathrm{~d}, J=3.6 \mathrm{~Hz}, 1 \mathrm{H}), 7.36(\mathrm{~m}, 1 \mathrm{H}), 7.14(\mathrm{~s}$, 1H), $7.11(\mathrm{~m}, 1 \mathrm{H}), 3.99(\mathrm{~s}, 1 \mathrm{H}), 3.95(\mathrm{~d}, J=3.4 \mathrm{~Hz}, 8 \mathrm{H}) .{ }^{13} \mathbf{C}$ NMR $\left(151 \mathrm{MHz}, \mathrm{CDCl}_{3}\right) \delta 191.2,153.6$, 143.6, 132.1, 131.7, 127.7, 125.8, 125.1, 106.8, 61.0, 56.3. HRMS (ESI-quadrupole) $\mathrm{m} / \mathrm{z}:[\mathrm{M}+\mathrm{H}]^{+}$calcd for $\mathrm{C}_{16} \mathrm{H}_{15} \mathrm{~N}_{3} \mathrm{O}_{4} \mathrm{~S}_{1} \mathrm{H}, 346.0855$; found, 346.0843 .

\section{Separation of atropoisomers of compound 7a by using Chiral HPLC}

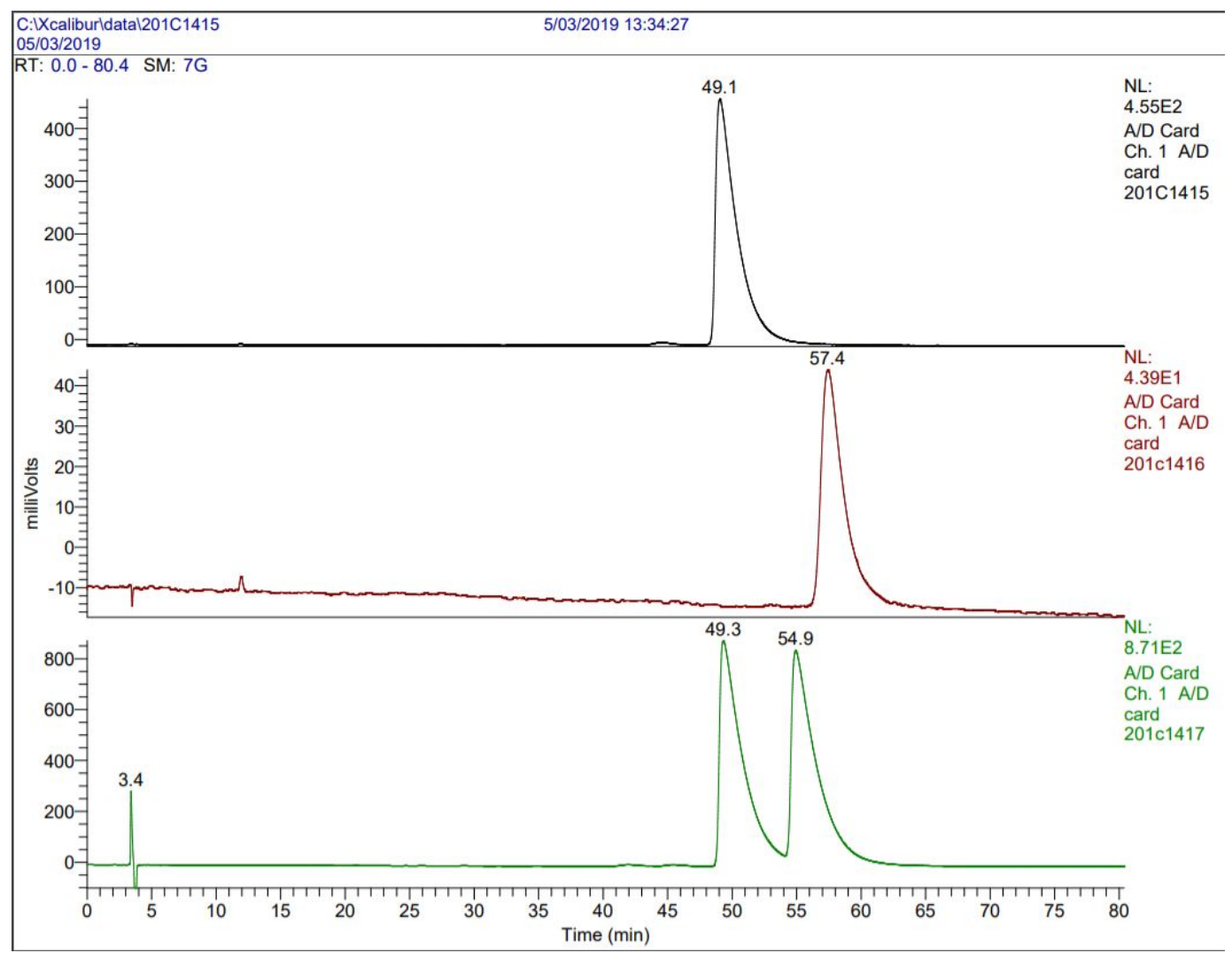

Figure S1. Device and method details for separation of atropoisomers. Device: HPLC system Waters Alliance 2695; detector Waters 2487 UV; Column: Analytic: Daicel Chiralpak IB, $4.6 \mathrm{~mm}$ x 250 mm, 5 $\mu \mathrm{L}$ Semi-prep: Daicel Chiralpak IB, $10 \mathrm{~mm}$ x $250 \mathrm{~mm}, 5 \mu \mathrm{L}$; Method: Solvent: heptane/ethylacetate (80/20), Flow: $1 \mathrm{ml} / \mathrm{min}$ (analytic), $5 \mathrm{ml} / \mathrm{min}$ (semi-prep).

\section{References}

(1) Thomas, J.; Jana, S.; John, J.; Liekens, S.; Dehaen, W.; A general metal-free route towards the synthesis of 1,2,3triazoles from readily available primary amines and ketones. Chem. Commun. 2016, 52, 2885-2888.

(2) Faucher, N.; Ambroise, Y.; Cintrat, J.-C.; Doris, E.; Pillon, F.; Rousseau, B.; Highly Chemoselective Hydrogenolysis of Iodoarenes. J. Org. Chem. 2002, 67, 932-934.

(3) Odlo, K.; Fournier-Dit-Chabert, J.; Ducki, S.; Gani, O. A. B. S. M.; Sylte, I.; Hansen, T. V.; 1,2,3-Triazole analogs of combretastatin A-4 as potential microtubule-binding agents. Bioorg. Med. Chem. 2010, 18, 6874-6885. 


\section{${ }^{1} \mathrm{H}$ and ${ }^{13} \mathrm{C}$ NMR spectra}

${ }^{1} \mathbf{H}$ NMR Spectra of (5) (300 MHz, $\left.\mathrm{CDCl}_{3}\right)$ :

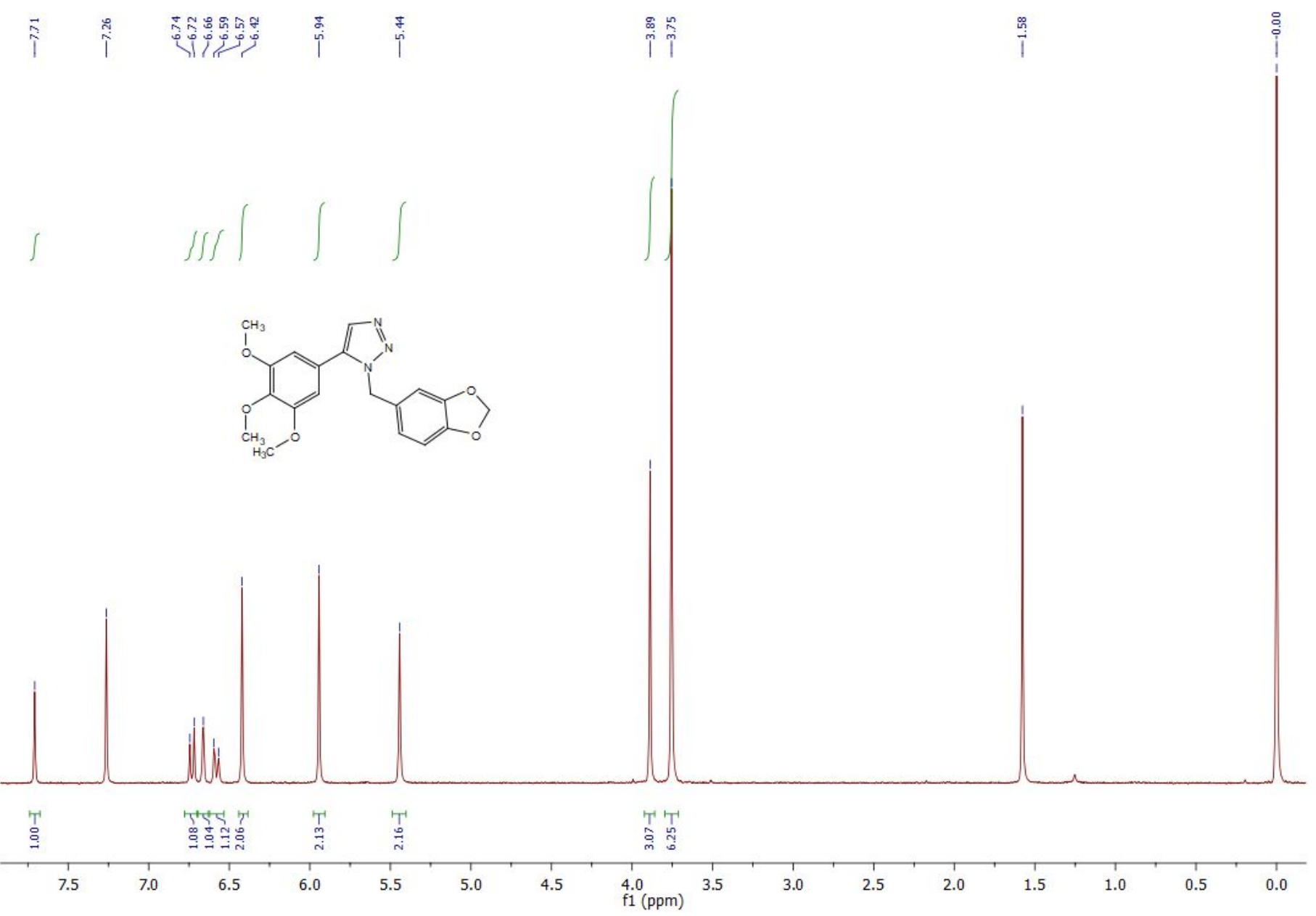


${ }^{13}$ C NMR Spectra of (5) (600 MHz, $\left.\mathrm{CDCl}_{3}\right)$ :

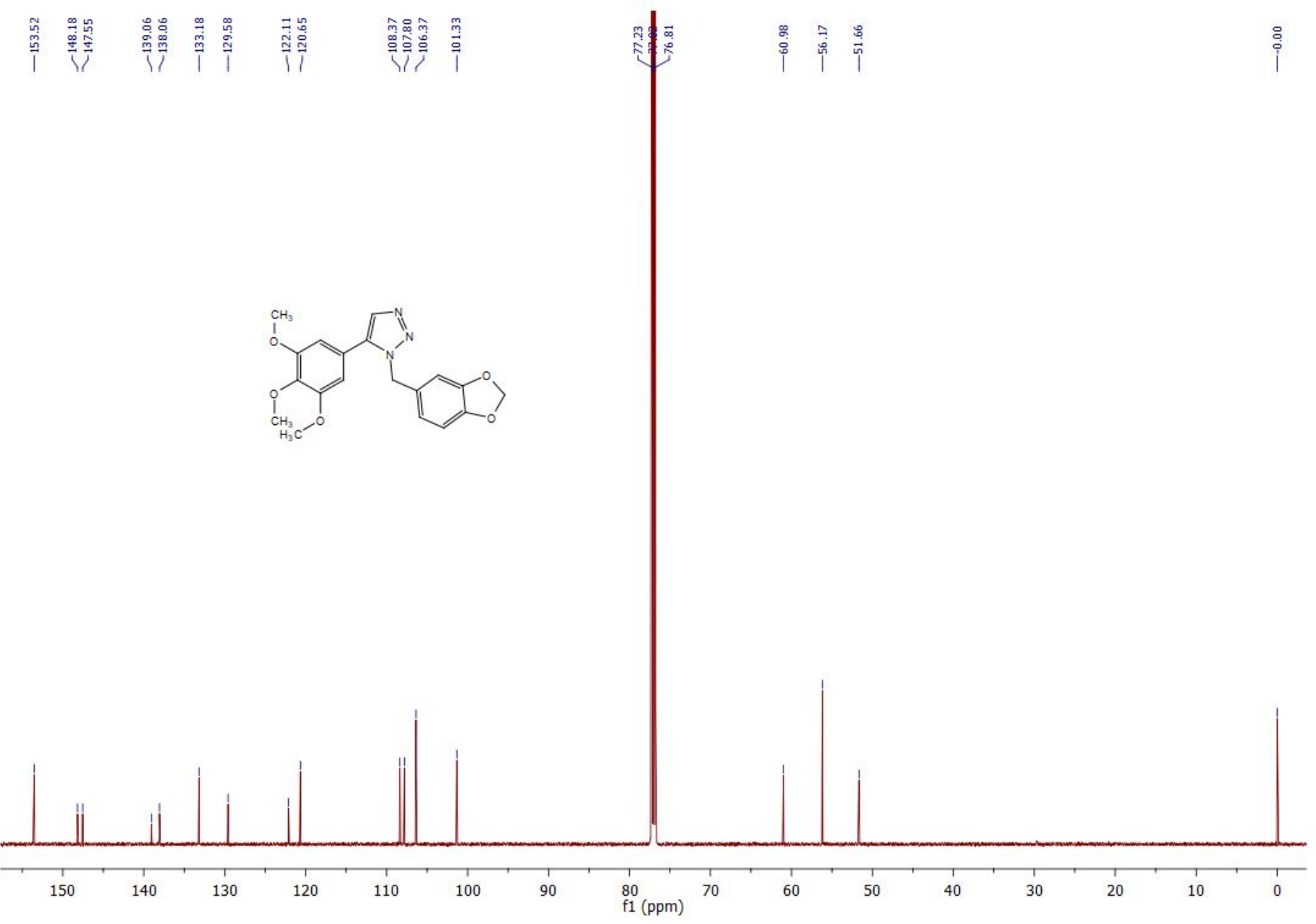


${ }^{\mathbf{1}} \mathbf{H}$ NMR Spectra of (6) (300 MHz, $\left.\mathrm{CDCl}_{3}\right)$ :

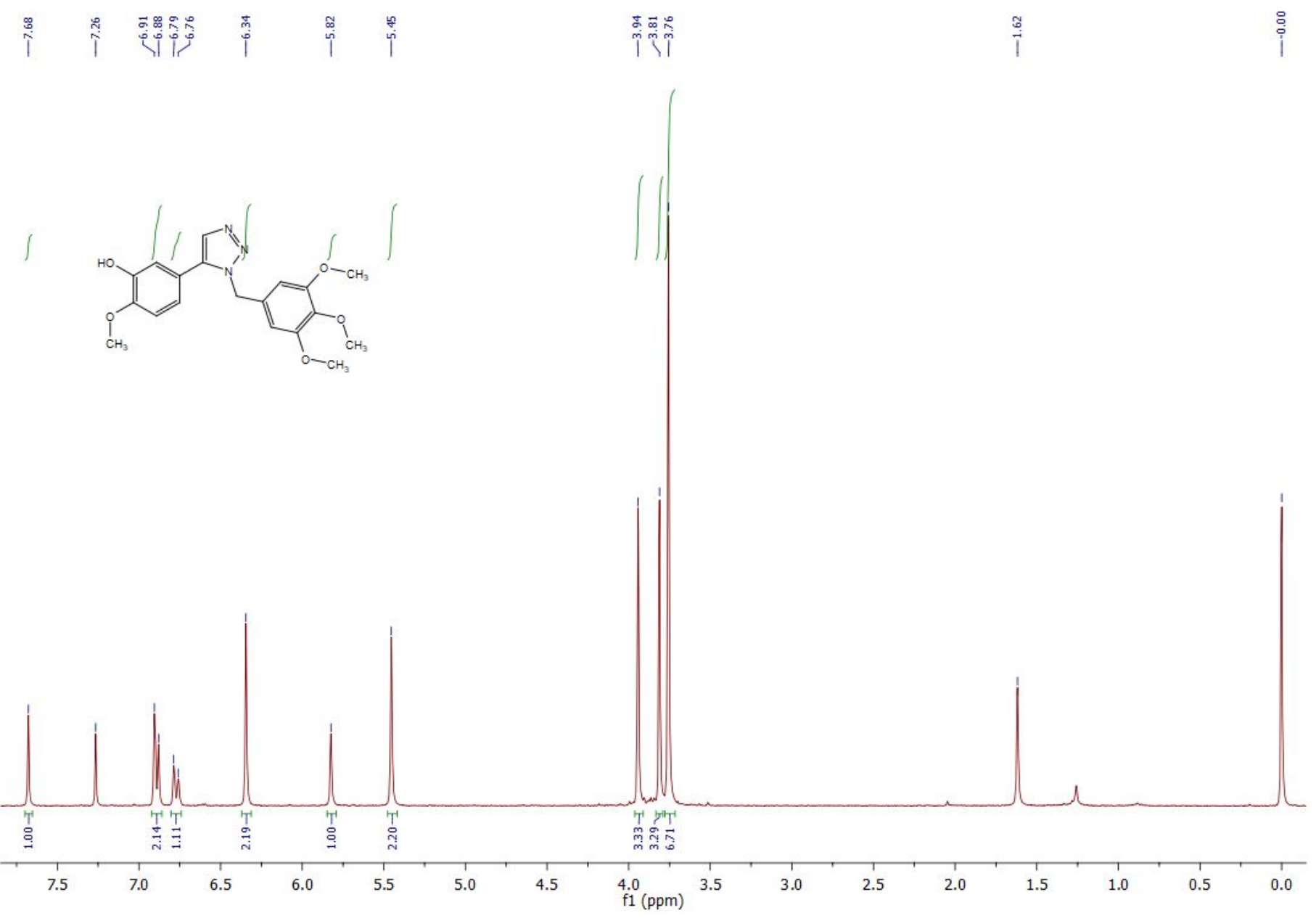


${ }^{13}$ C NMR Spectra of (6) (300 MHz, $\left.\mathrm{CDCl}_{3}\right)$ :

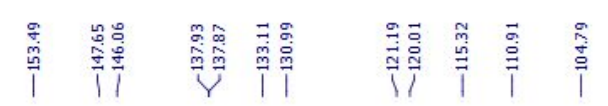<smiles>COc1ccc(-c2cnnn2CC2CC(OC)C(OC)C(OC)C2)cc1O</smiles>

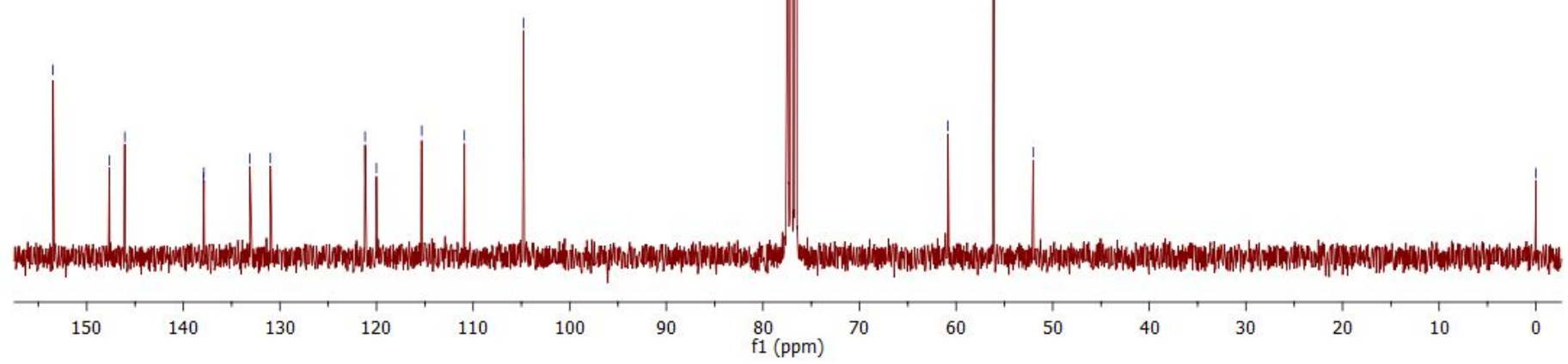


${ }^{\mathbf{1}} \mathbf{H}$ NMR Spectra of (7) (300 MHz, $\left.\mathrm{CDCl}_{3}\right)$ :

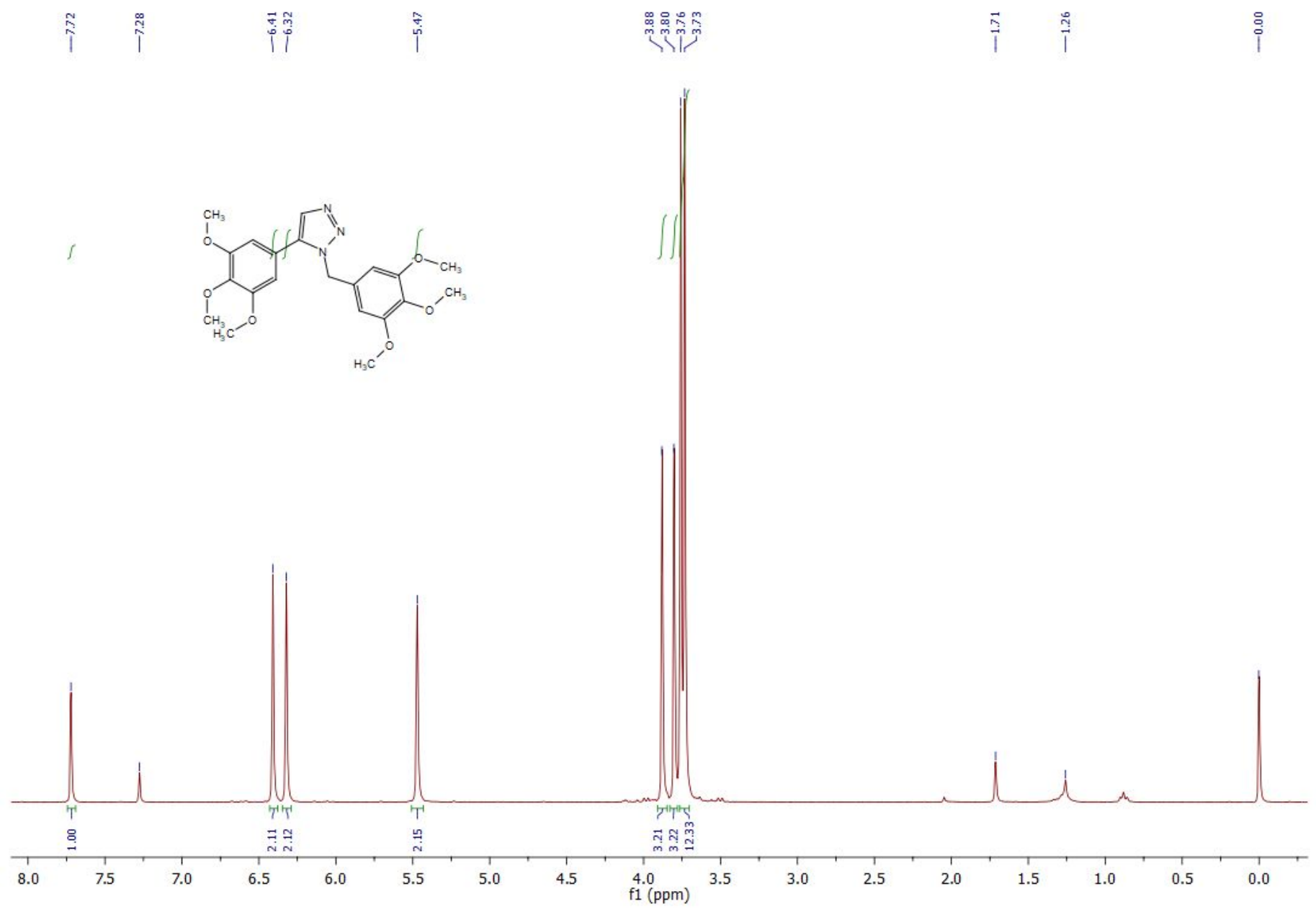


${ }^{13}$ C NMR Spectra of (7) (300 MHz, $\left.\mathrm{CDCl}_{3}\right)$ :

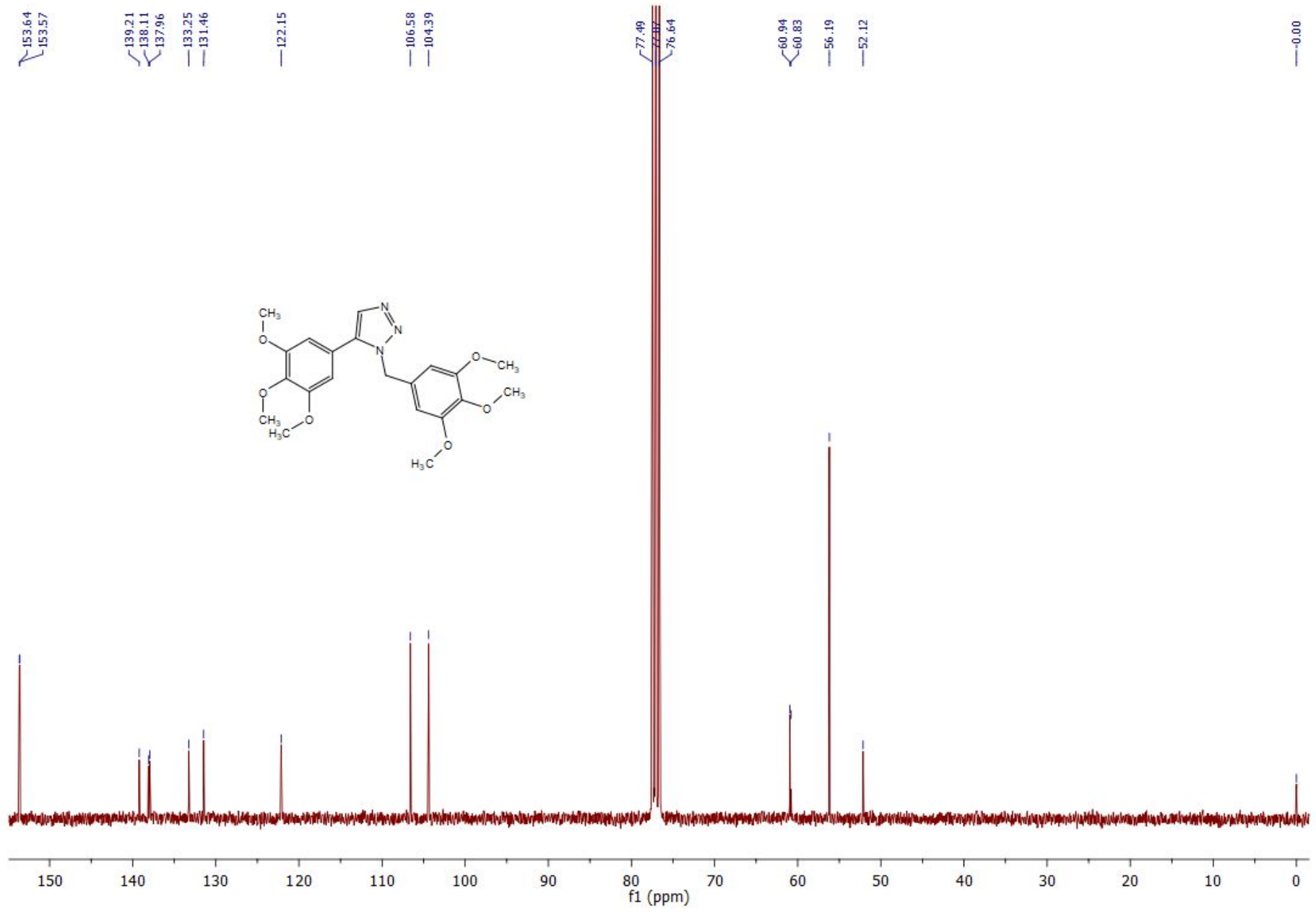


${ }^{1}$ H NMR Spectra of (8) (300 MHz, $\left.\mathrm{CDCl}_{3}\right)$ :

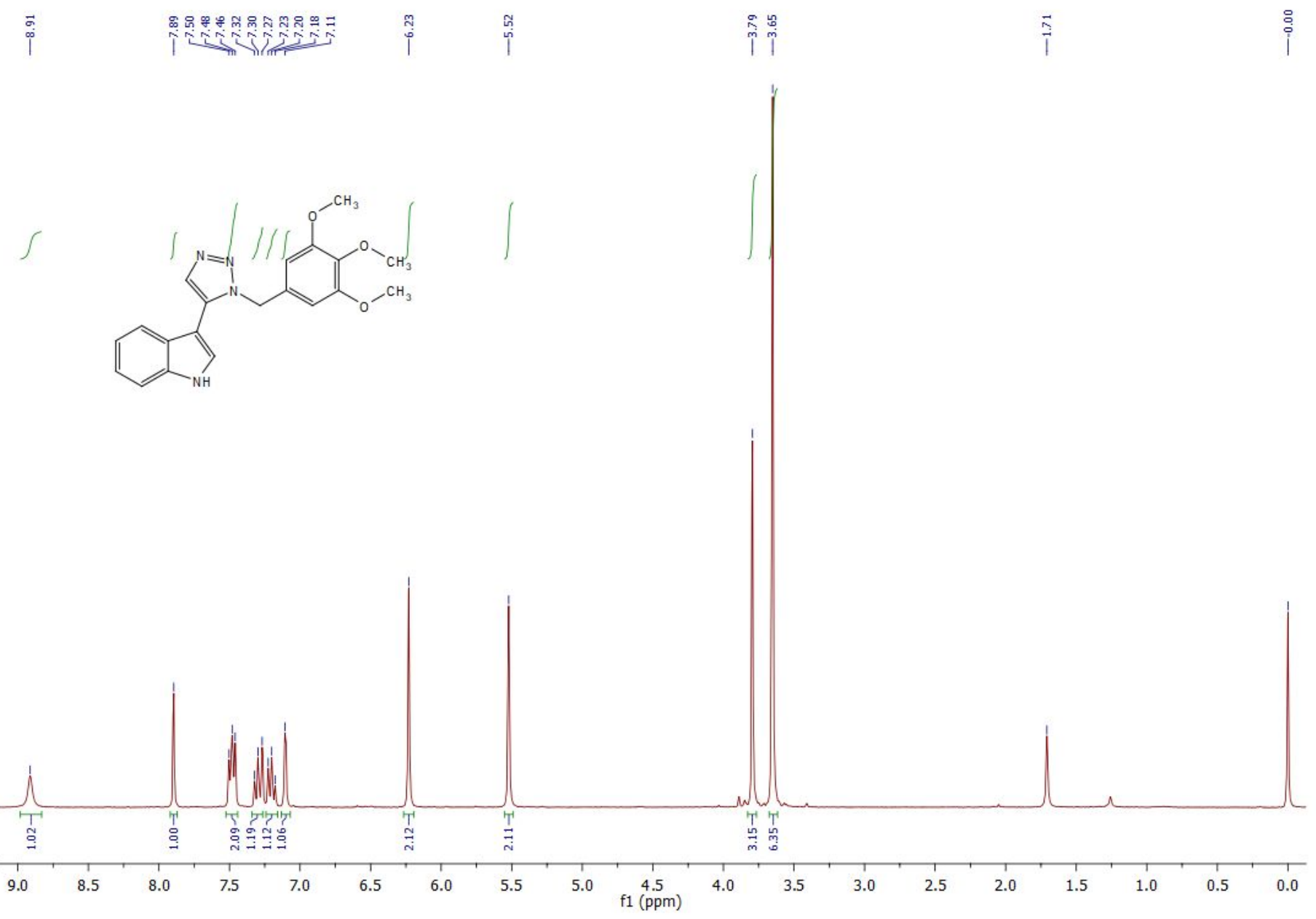


${ }^{13}$ C NMR Spectra of (8) (300 MHz, $\left.\mathrm{CDCl}_{3}\right)$ :

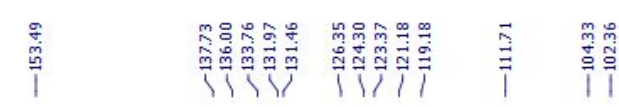
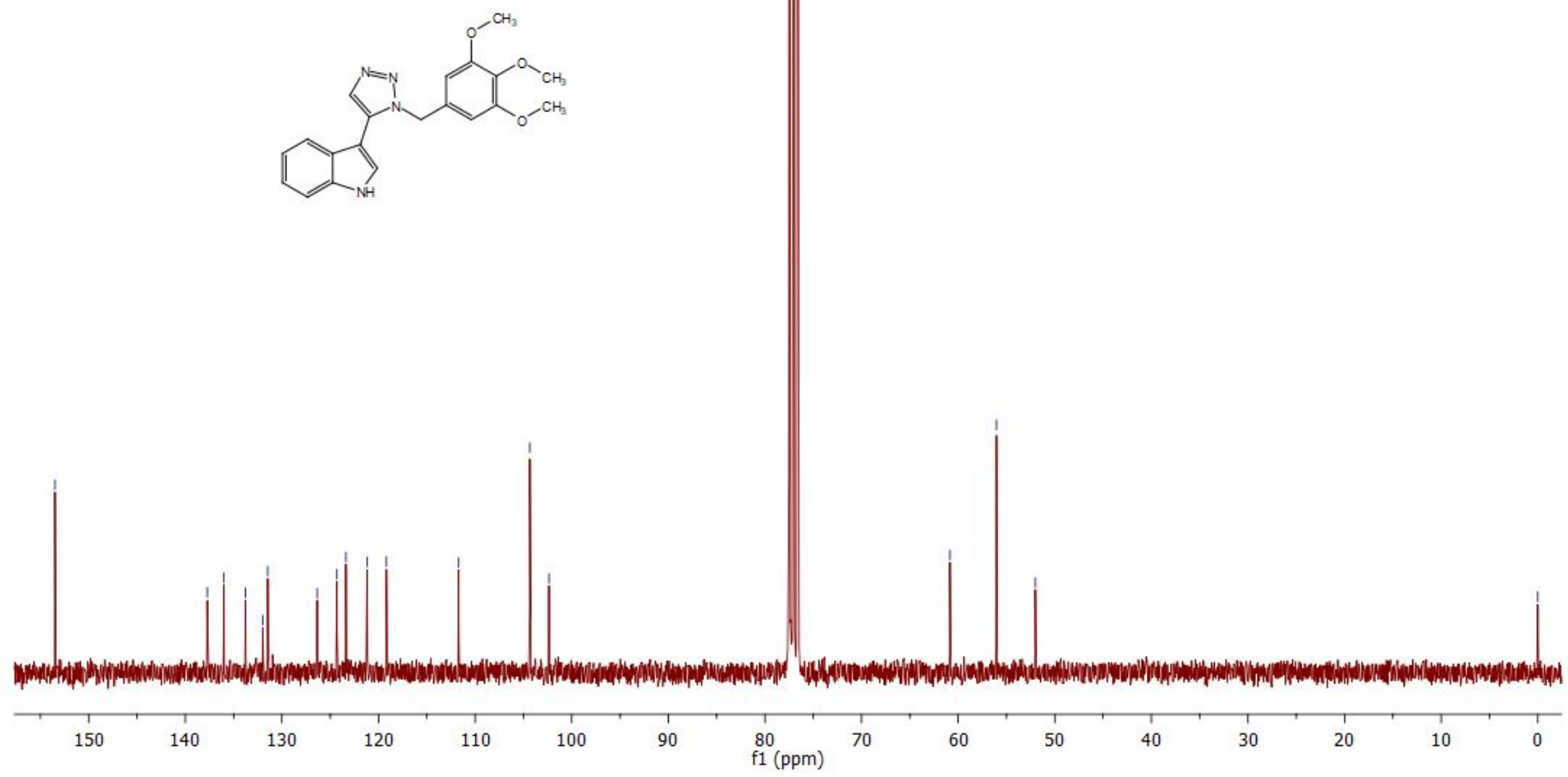
${ }^{1}$ H NMR Spectra of (9) (300 MHz, $\mathrm{CDCl}_{3}$ ):
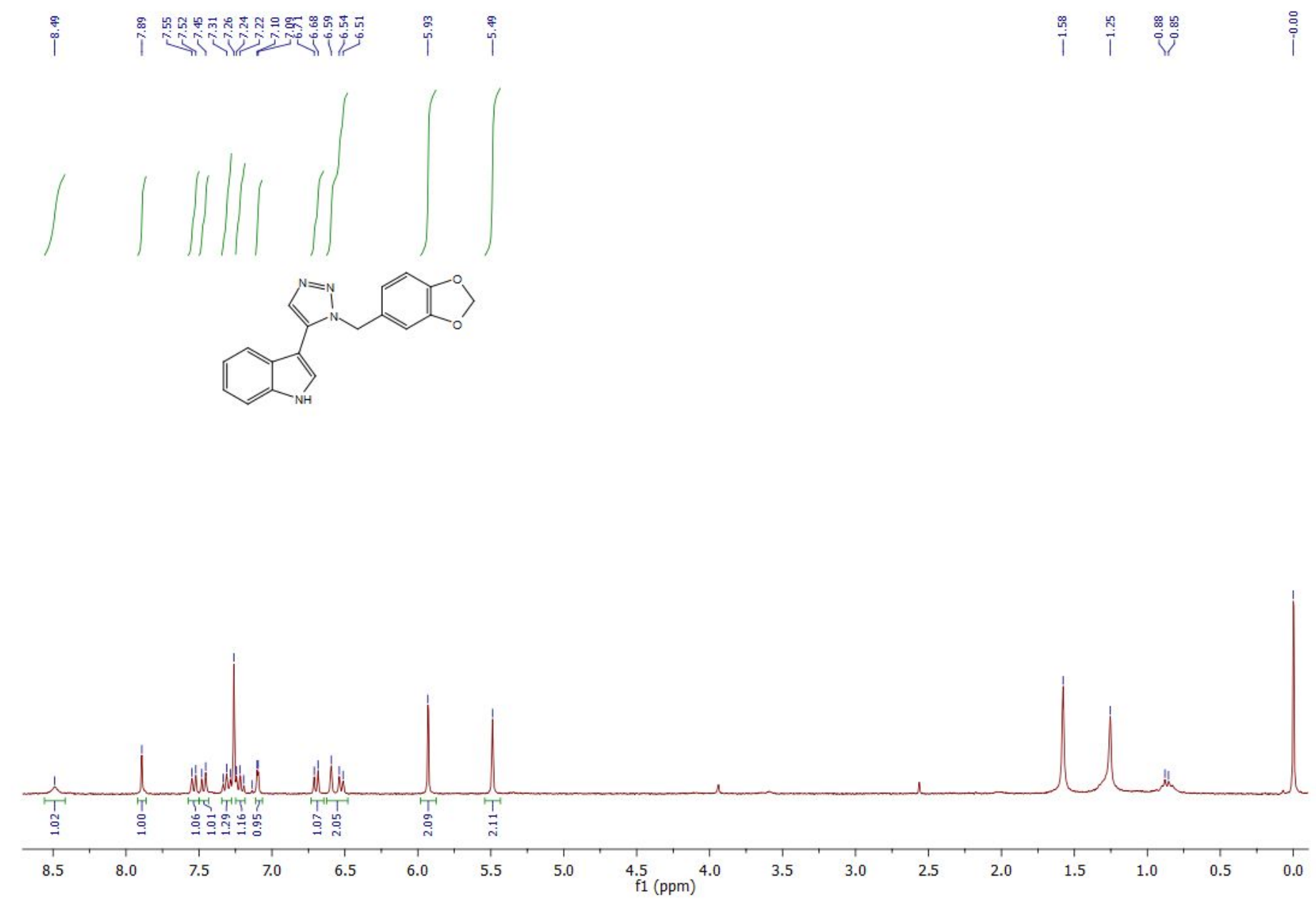
${ }^{13}$ C NMR Spectra of (9) (600 MHz, $\left.\mathrm{CDCl}_{3}\right)$ :

ll

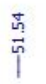

$\stackrel{8}{i}$<smiles>c1ccc2c(-c3cnnn3Cc3ccc4c(c3)OCO4)c[nH]c2c1</smiles>

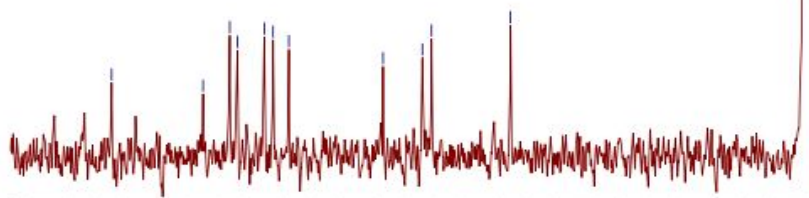

$\begin{array}{lllllllllllll}140 & 135 & 130 & 125 & 120 & 115 & 110 & 105 & 100 & 95 & 90 & 85 & 80\end{array}$ $\begin{array}{ccc}75 & 70 & 65 \\ \mathrm{f} 1(\mathrm{ppm}) & \end{array}$ 
${ }^{\mathbf{1}} \mathbf{H}$ NMR Spectra of (10) (300 MHz, $\left.\mathrm{CDCl}_{3}\right)$ :

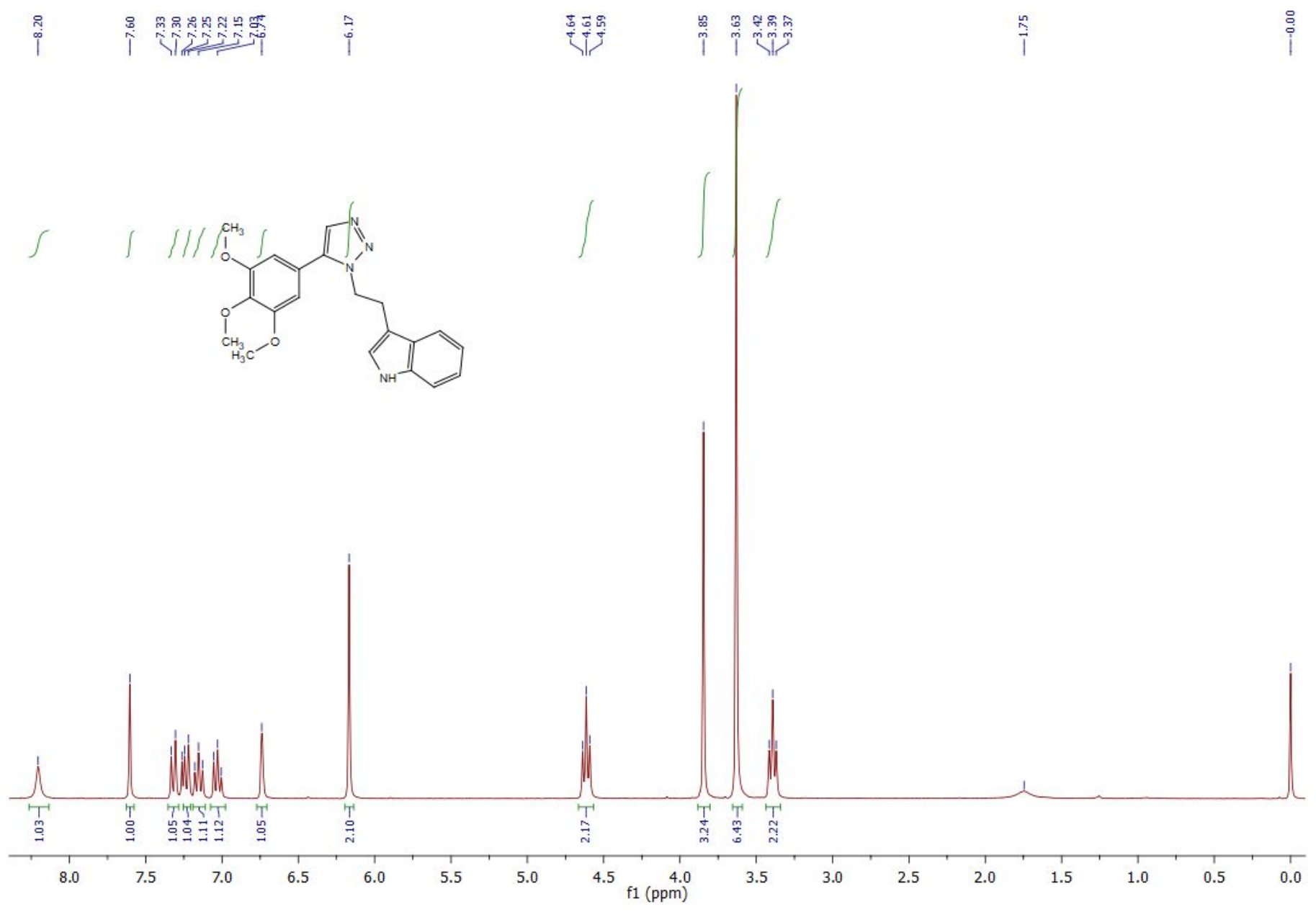


${ }^{13}$ C NMR Spectra of (10) (300 MHz, $\left.\mathrm{CDCl}_{3}\right)$ :

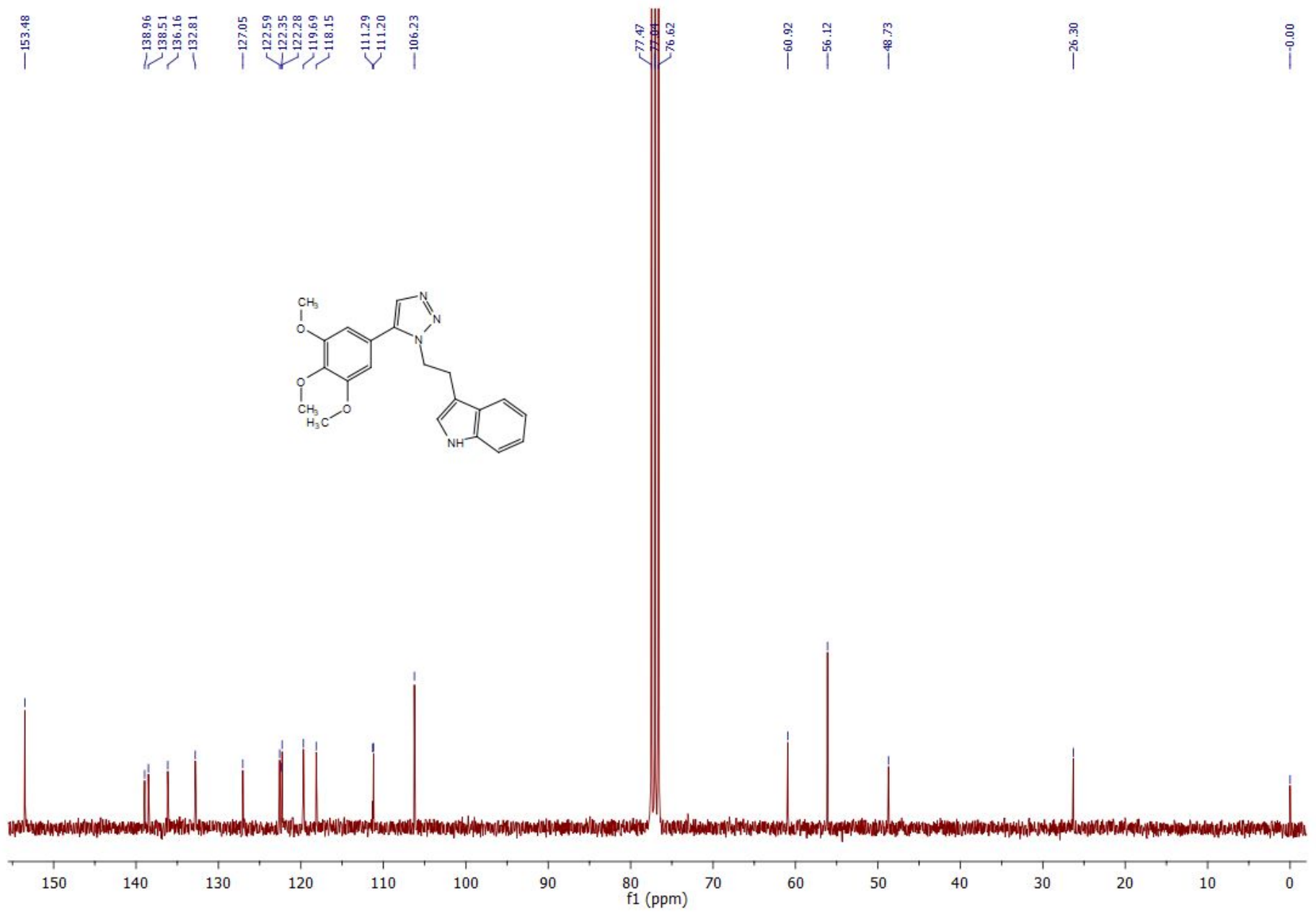


${ }^{\mathbf{1}} \mathbf{H}$ NMR Spectra of (11) (300 MHz, $\left.\mathrm{CDCl}_{3}\right)$ :

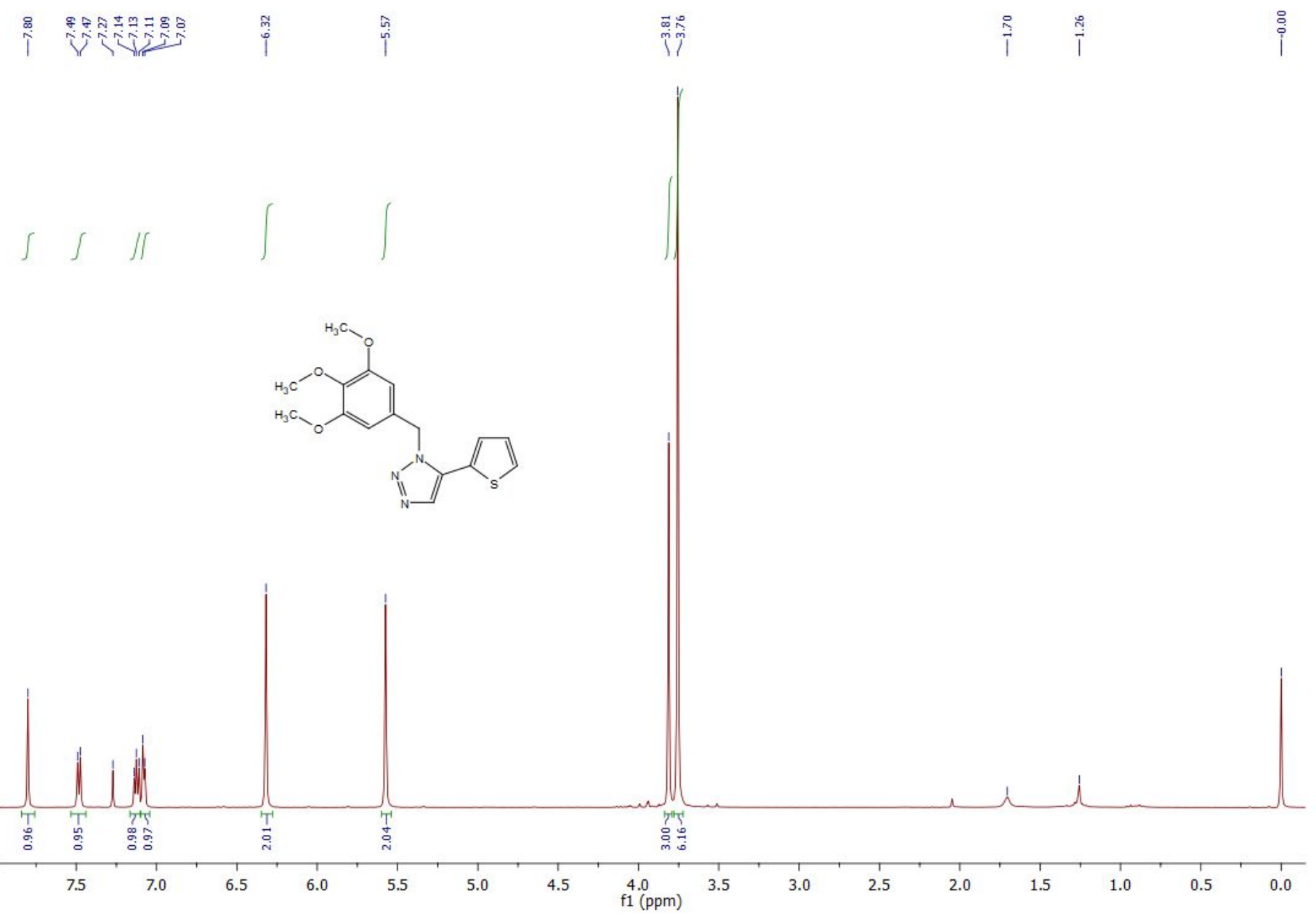


${ }^{13}$ C NMR Spectra of (11) (300 MHz, $\left.\mathrm{CDCl}_{3}\right)$ :

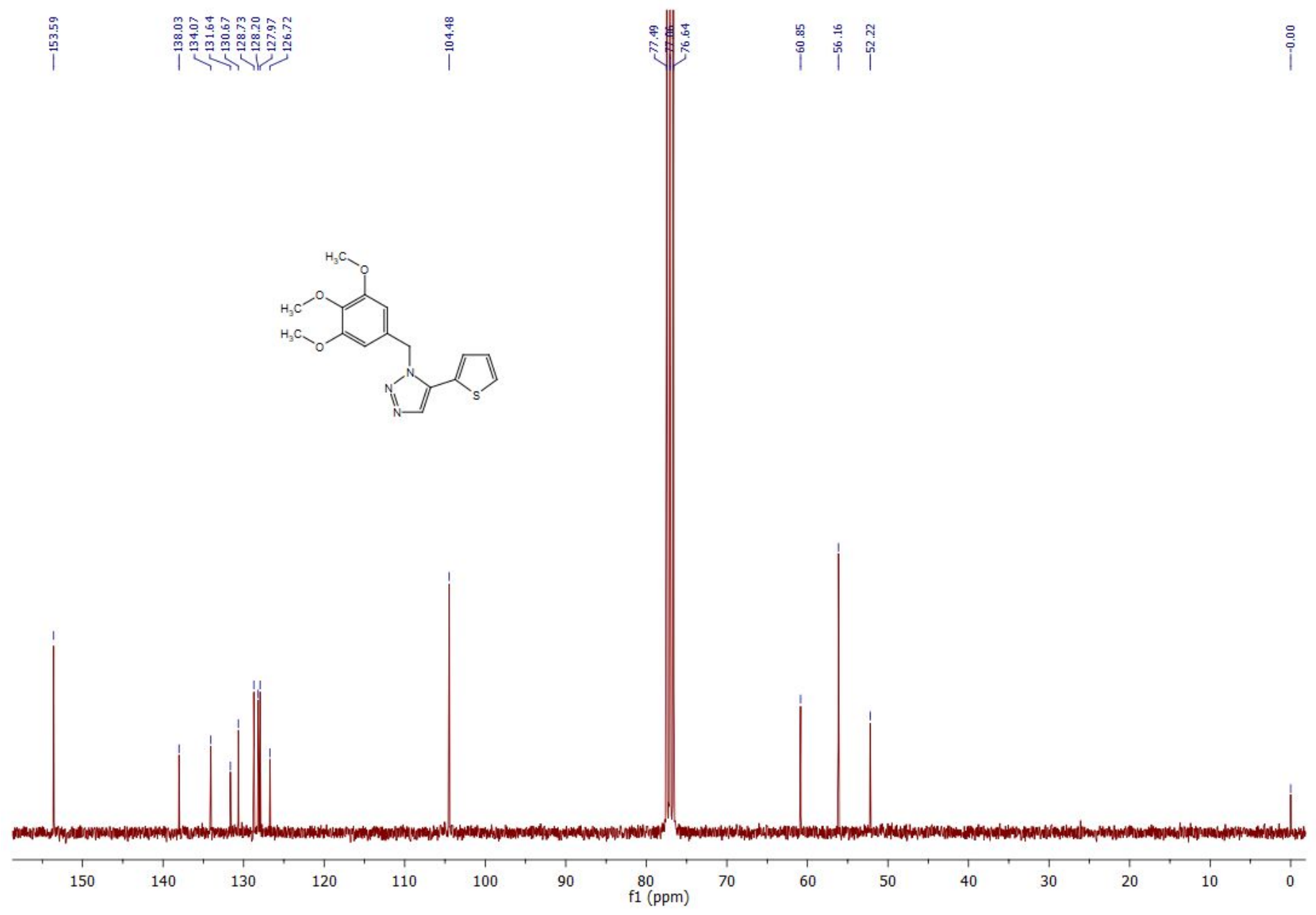


${ }^{1}$ H NMR Spectra of (12) (300 MHz, $\left.\mathrm{CDCl}_{3}\right)$ :

i.

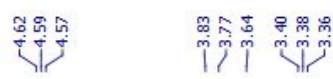

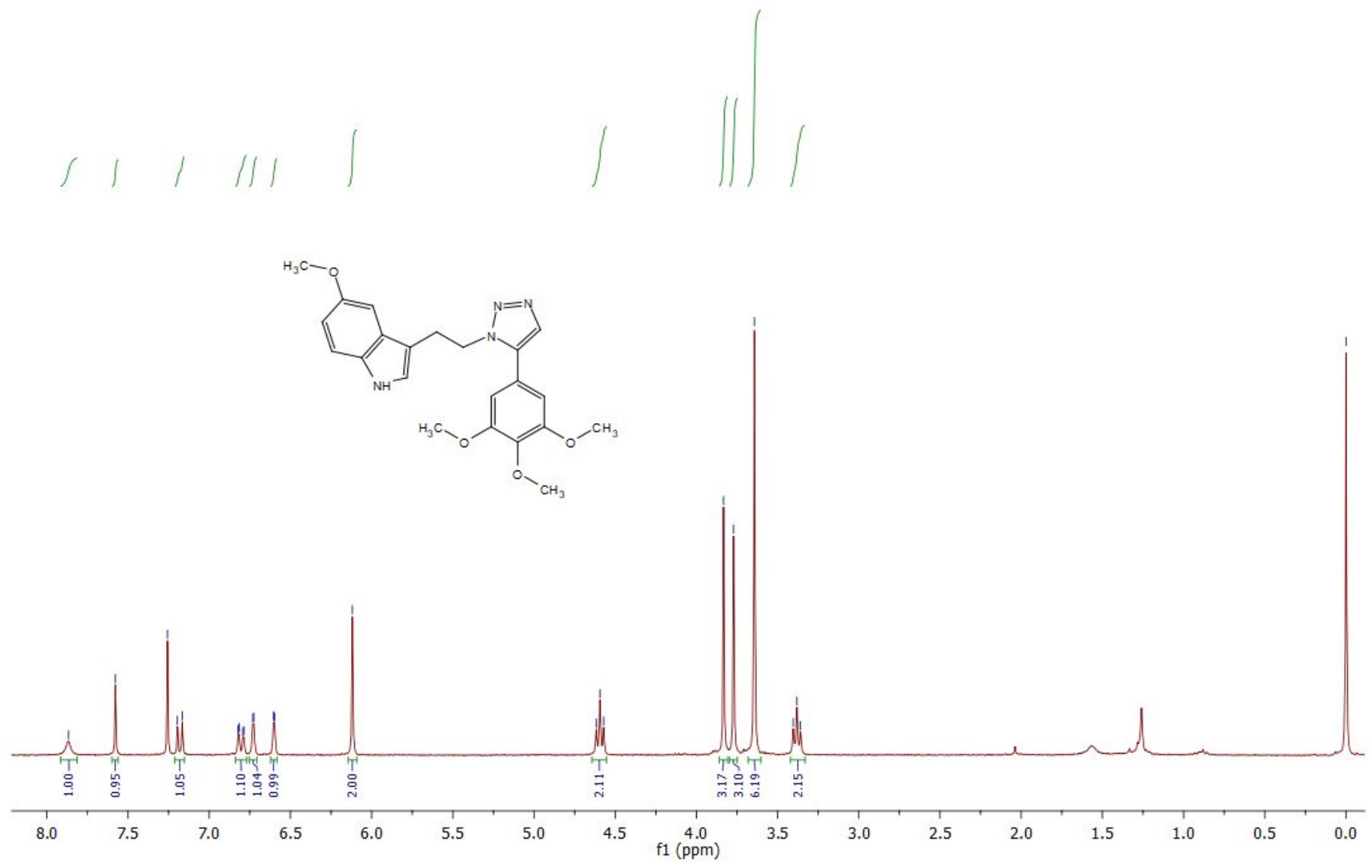


${ }^{13}$ C NMR Spectra of (12) (400 MHz, $\left.\mathrm{CDCl}_{3}\right)$ :

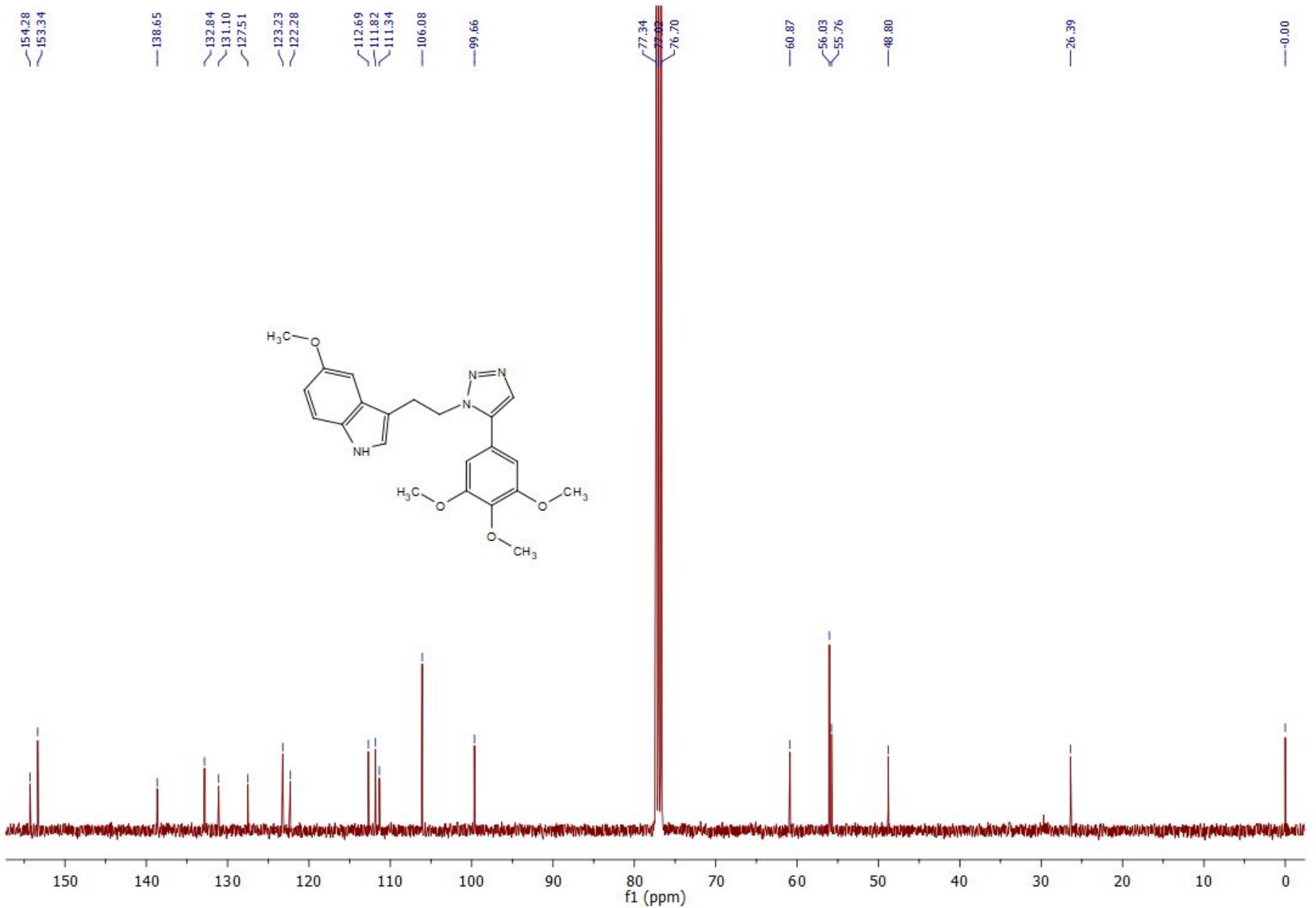


${ }^{\mathbf{1}} \mathbf{H}$ NMR Spectra of (13) (300 MHz, $\left.\mathrm{CDCl}_{3}\right)$ :

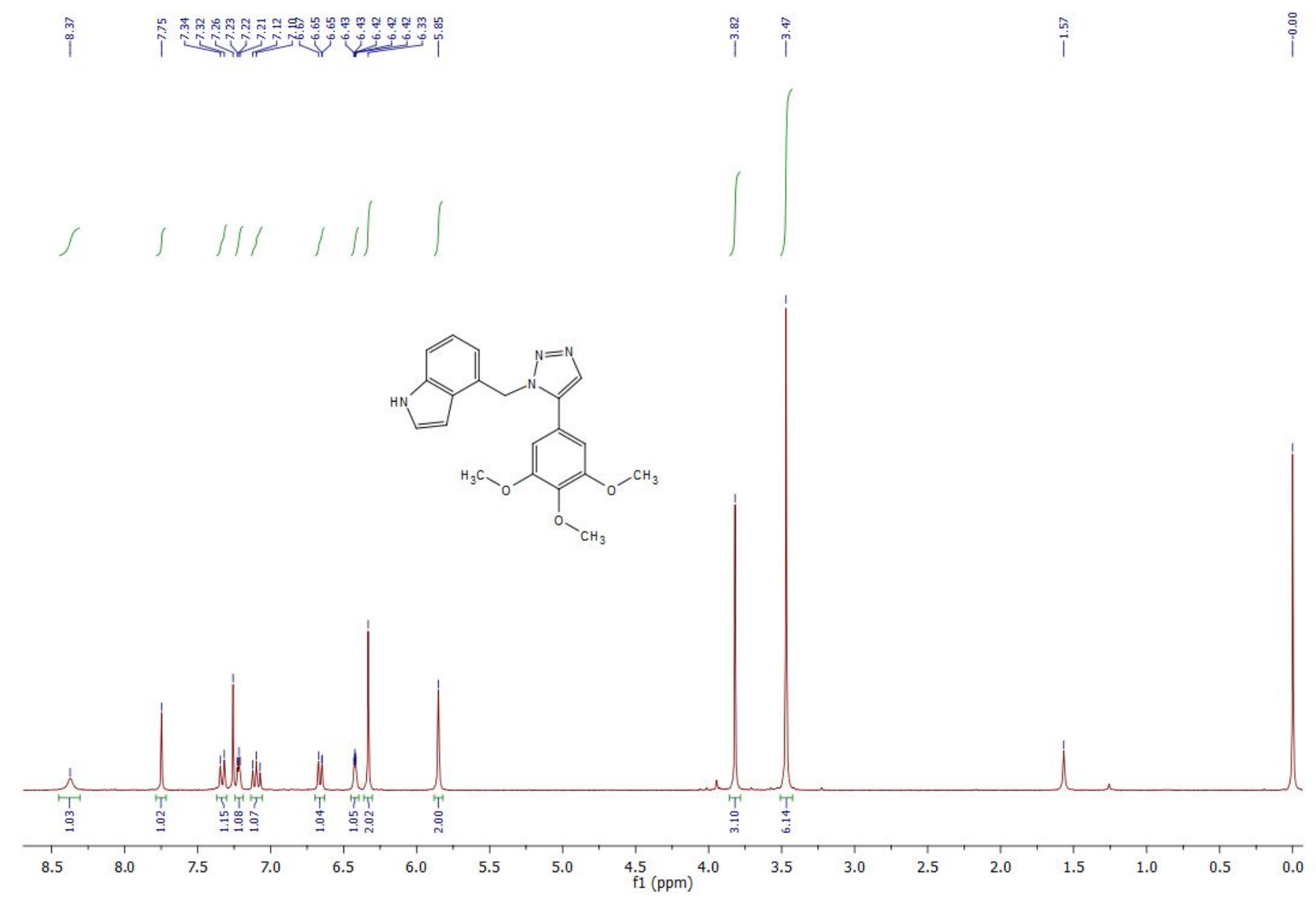


${ }^{13}$ C NMR Spectra of (13) (400 MHz, $\left.\mathrm{CDCl}_{3}\right)$ :

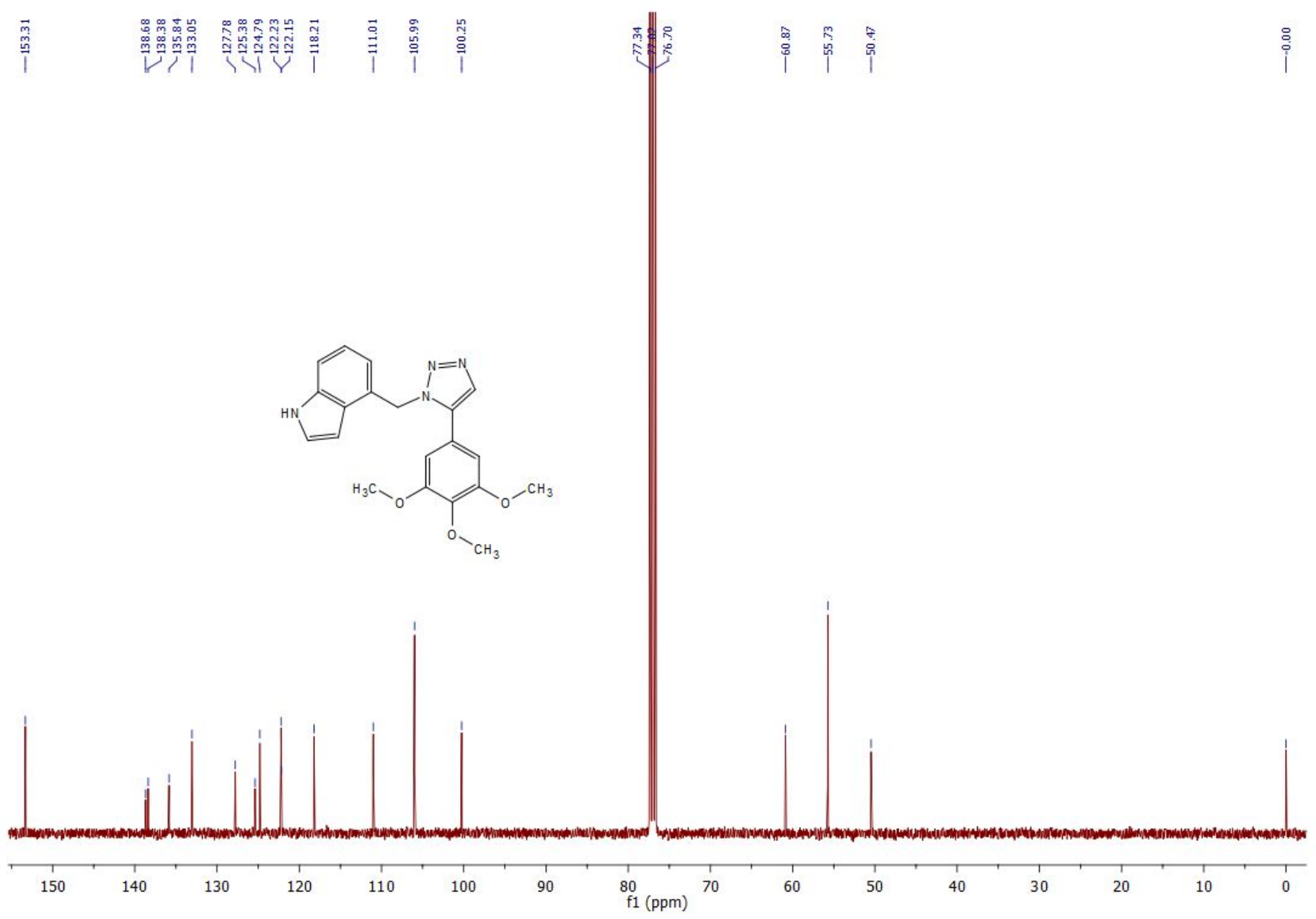


${ }^{\mathbf{1}} \mathbf{H}$ NMR Spectra of (14) (300 MHz, $\left.\mathrm{CDCl}_{3}\right)$ :

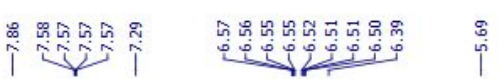

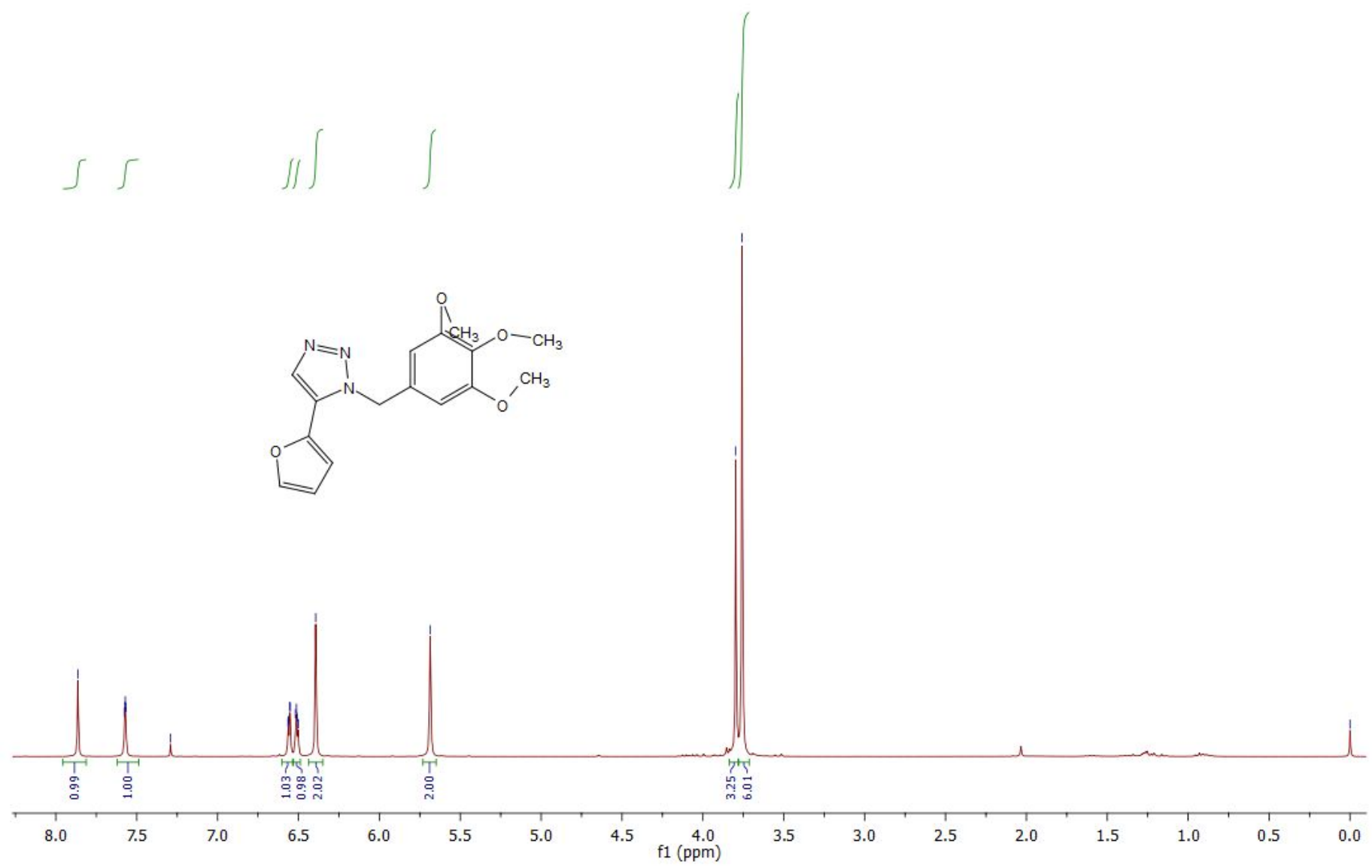


${ }^{13}$ C NMR Spectra of (14) (400 MHz, $\left.\mathrm{CDCl}_{3}\right)$ :

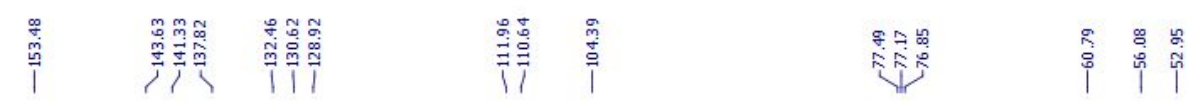

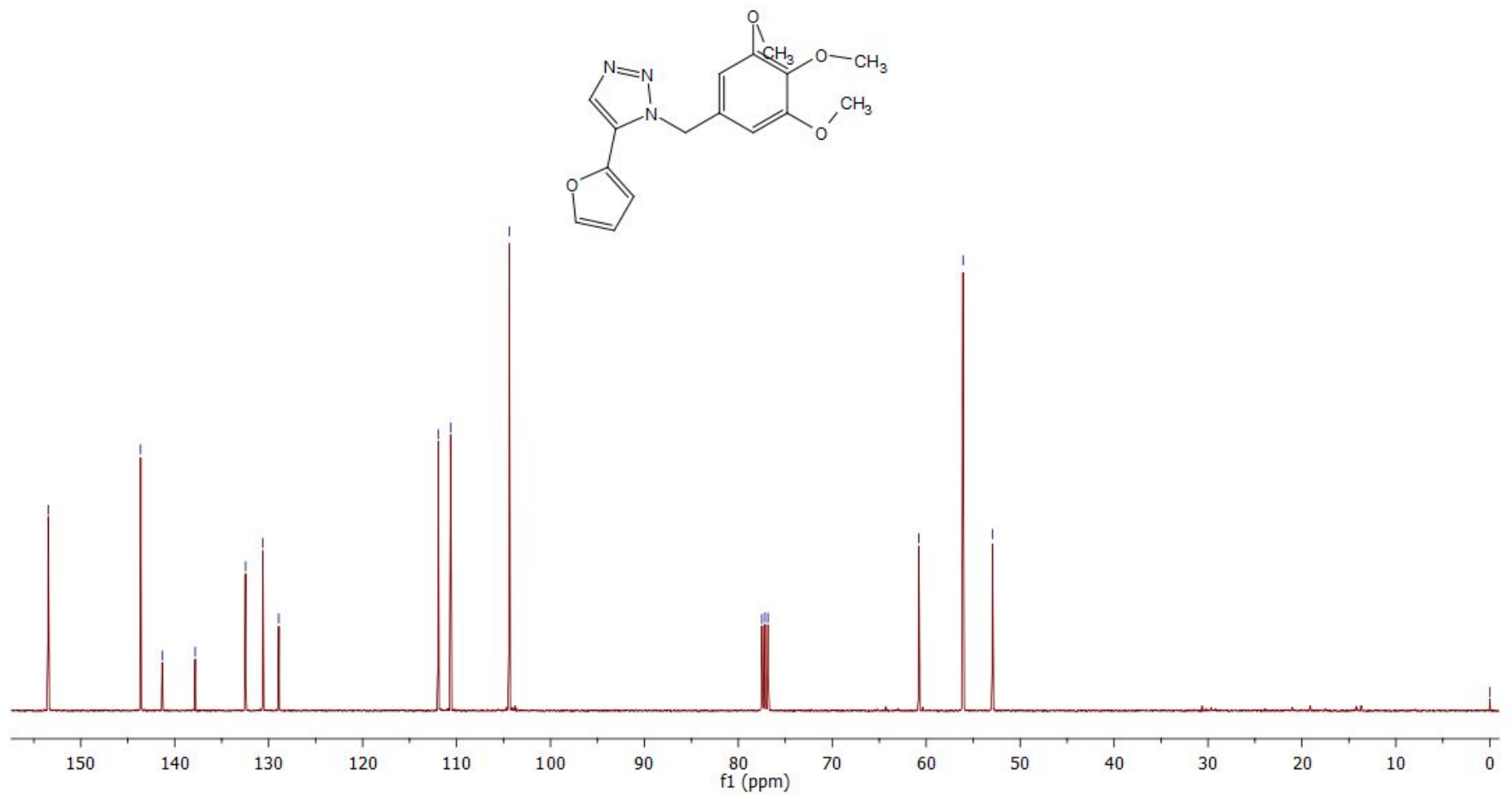


${ }^{\mathbf{1}} \mathbf{H}$ NMR Spectra of (15) (300 MHz, $\left.\mathrm{CDCl}_{3}\right)$ :

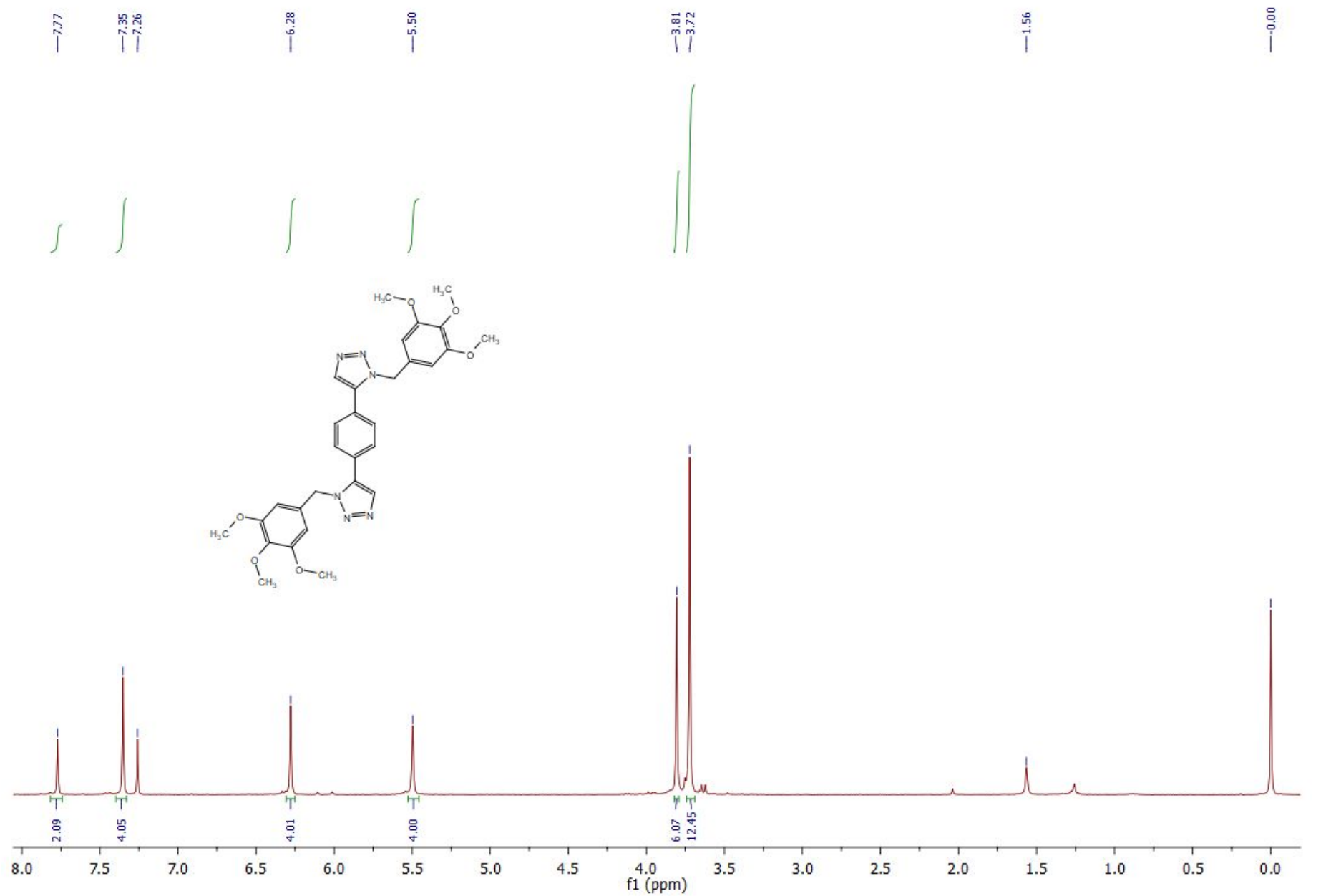


${ }^{13}$ C NMR Spectra of (15) (400 MHz, $\left.\mathrm{CDCl}_{3}\right)$ :

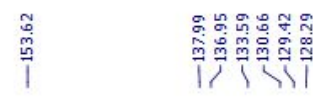

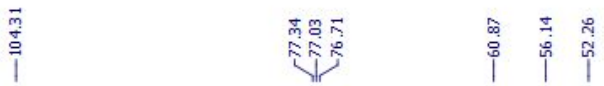

$\stackrel{0}{i}$
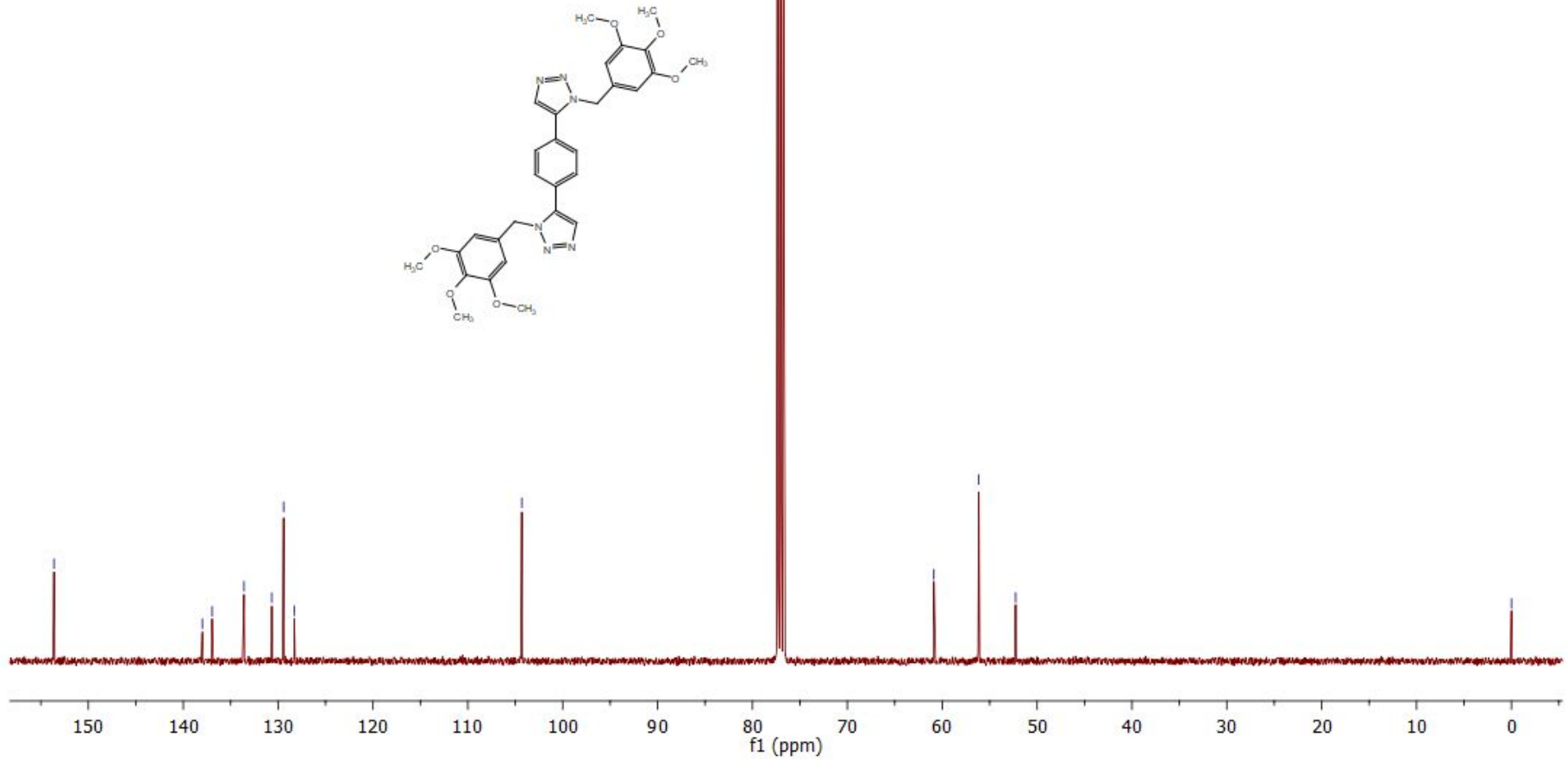
${ }^{1} \mathbf{H}$ NMR Spectra of (16) (300 MHz, $\left.\mathrm{CDCl}_{3}\right)$ :

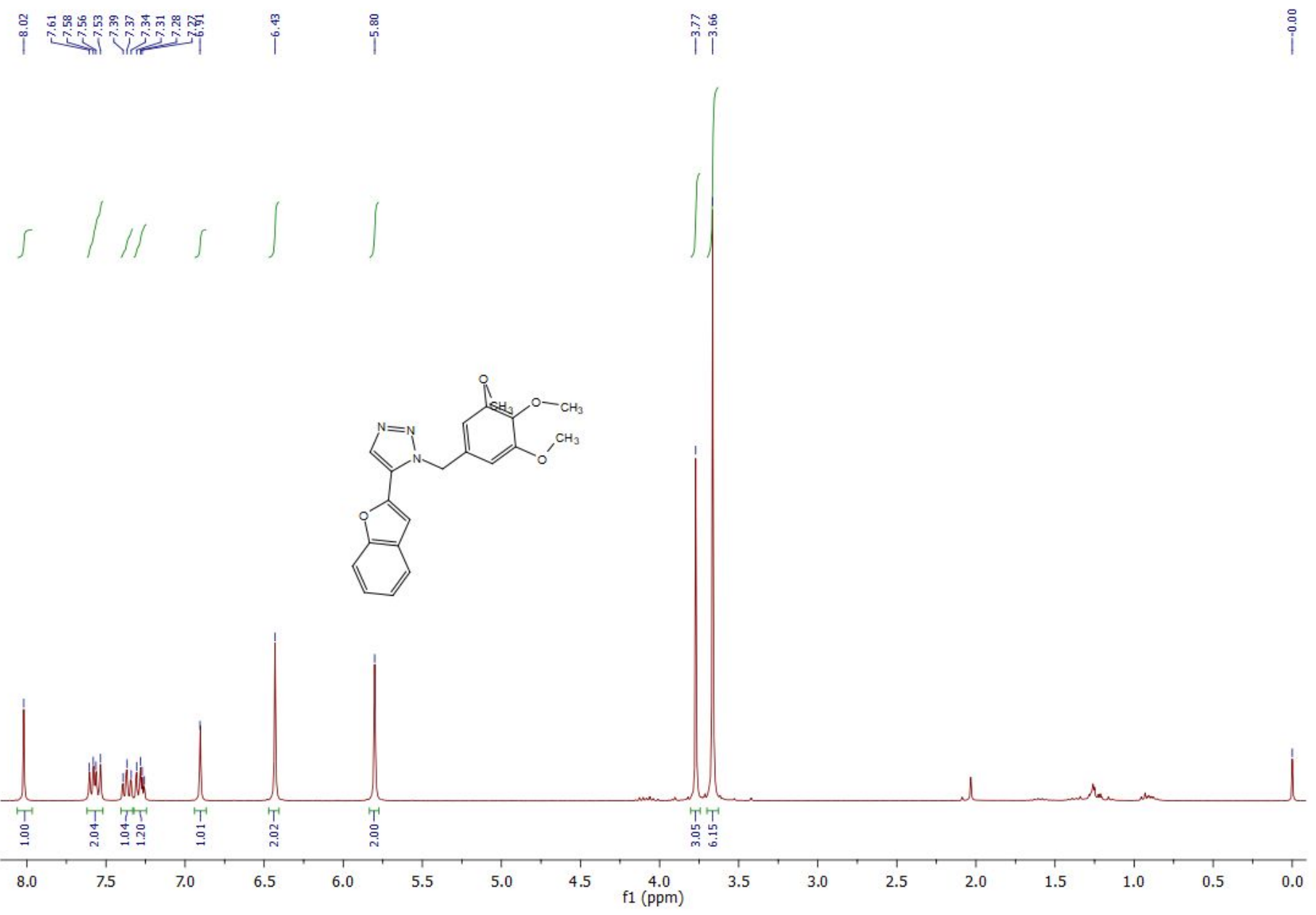


${ }^{13}$ C NMR Spectra of (16) (400 MHz, $\left.\mathrm{CDCl}_{3}\right)$ :

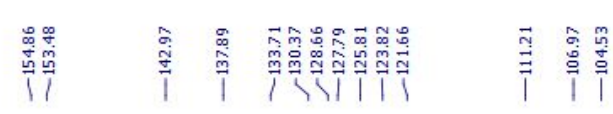

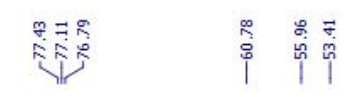
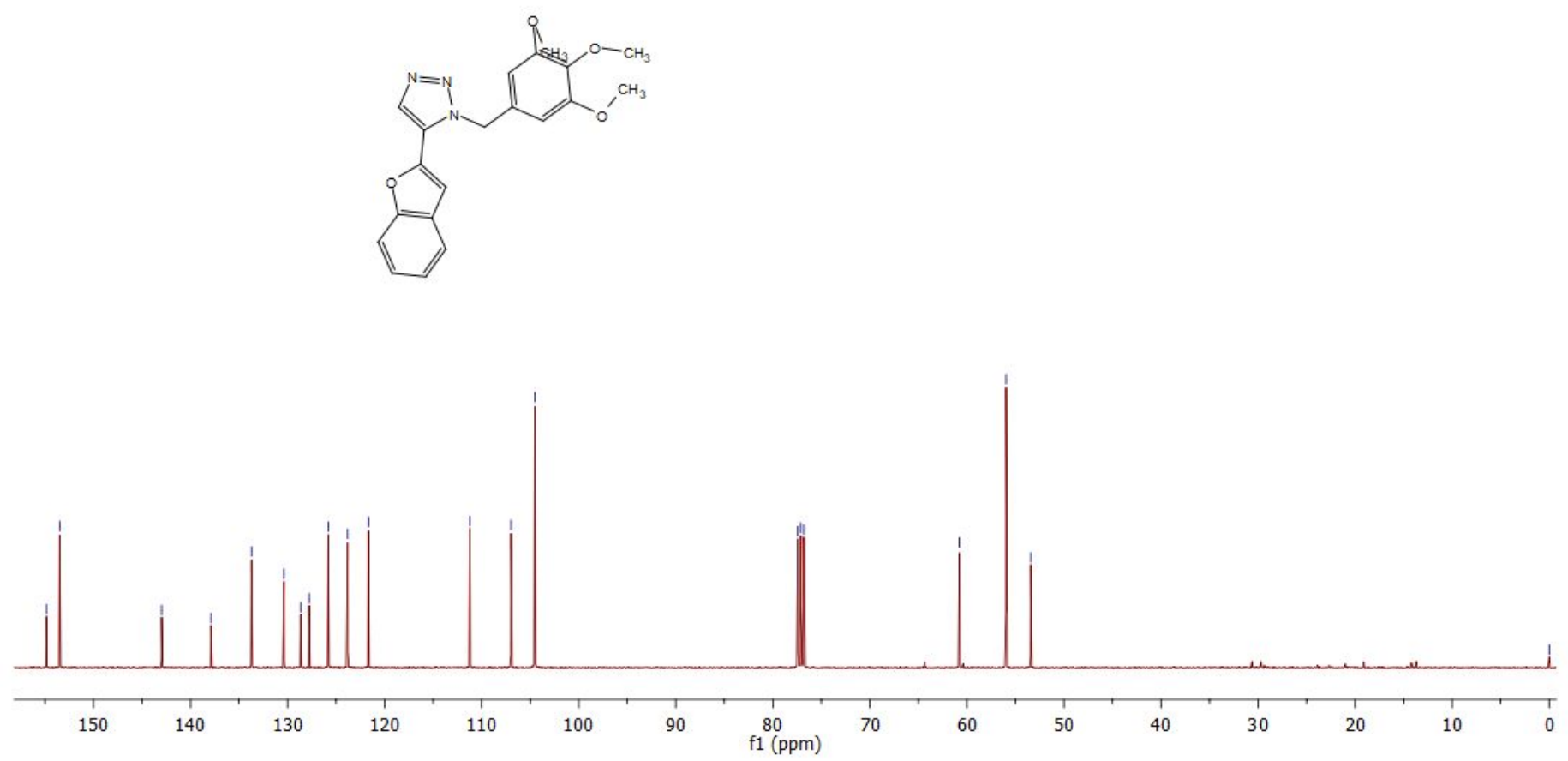
${ }^{\mathbf{1}} \mathbf{H}$ NMR Spectra of (5a) (300 MHz, $\left.\mathrm{CDCl}_{3}\right)$ :

i

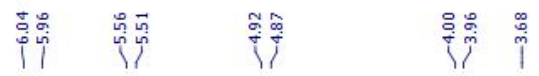

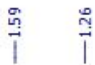

i

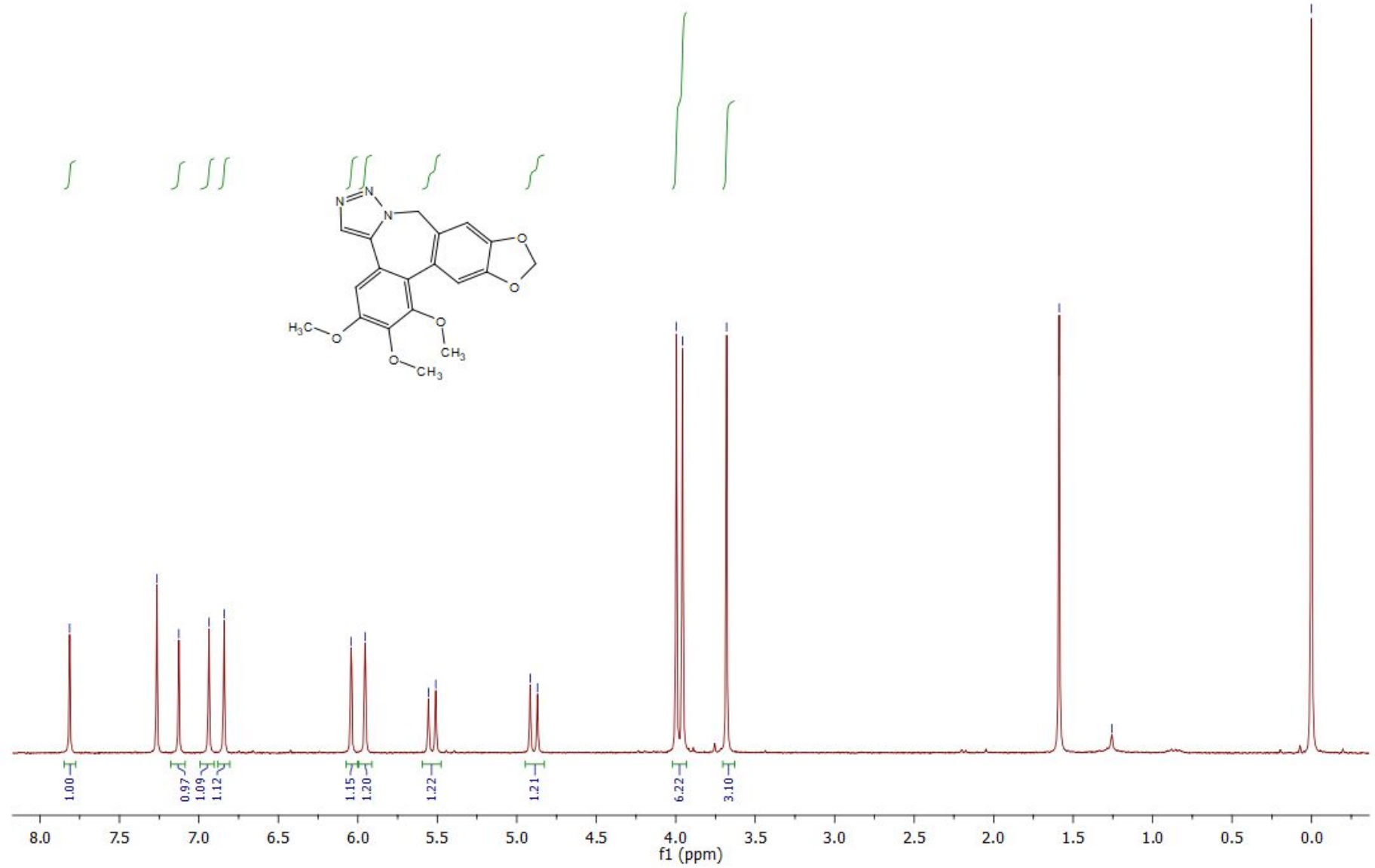


${ }^{13}$ C NMR Spectra of (5a) (600 MHz, $\left.\mathrm{CDCl}_{3}\right)$ :

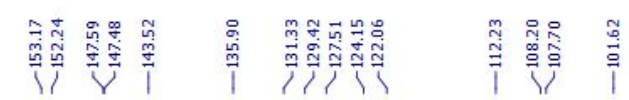
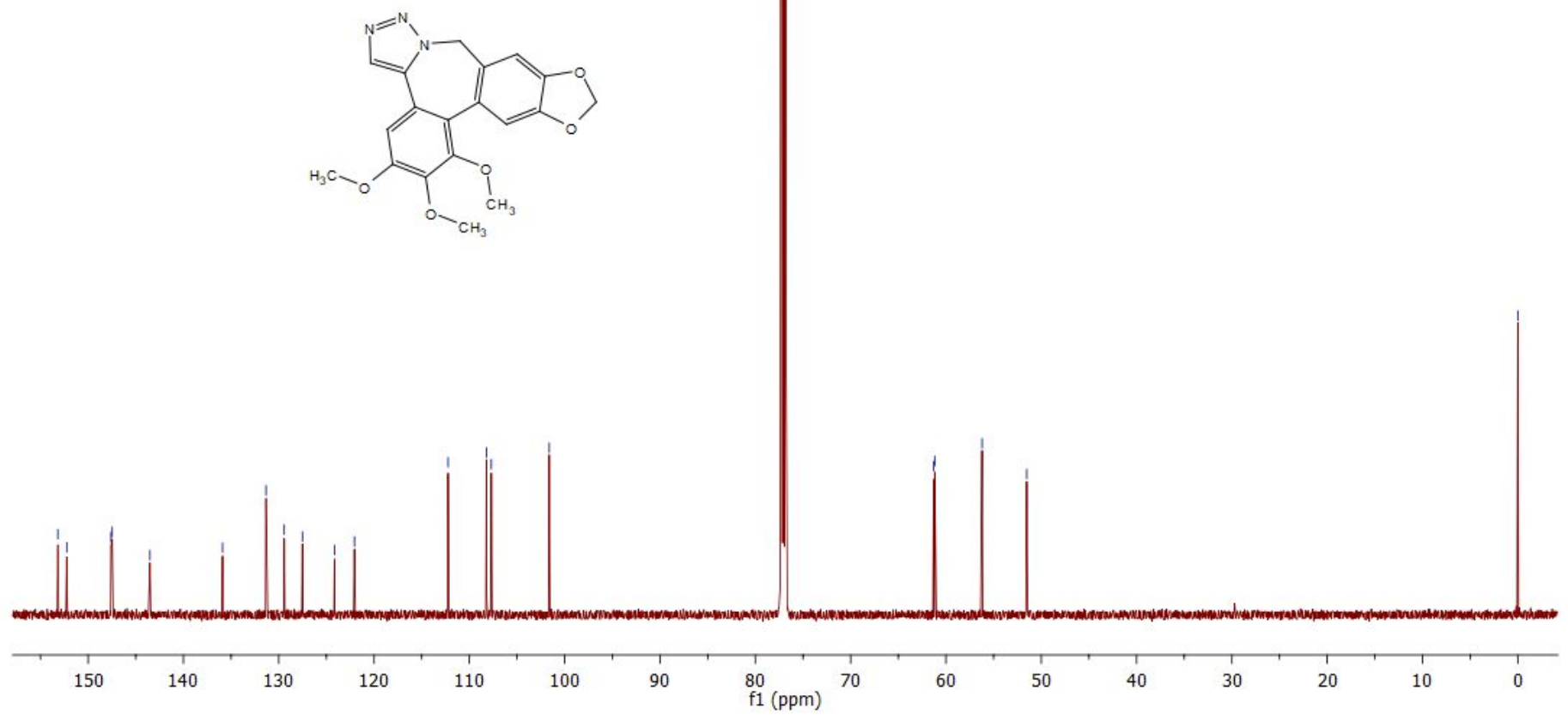
${ }^{1} \mathbf{H}$ NMR Spectra of (6a) (600 MHz, $\left.\mathrm{C}_{2} \mathrm{D}_{2} \mathrm{Cl}_{4}\right)$ :

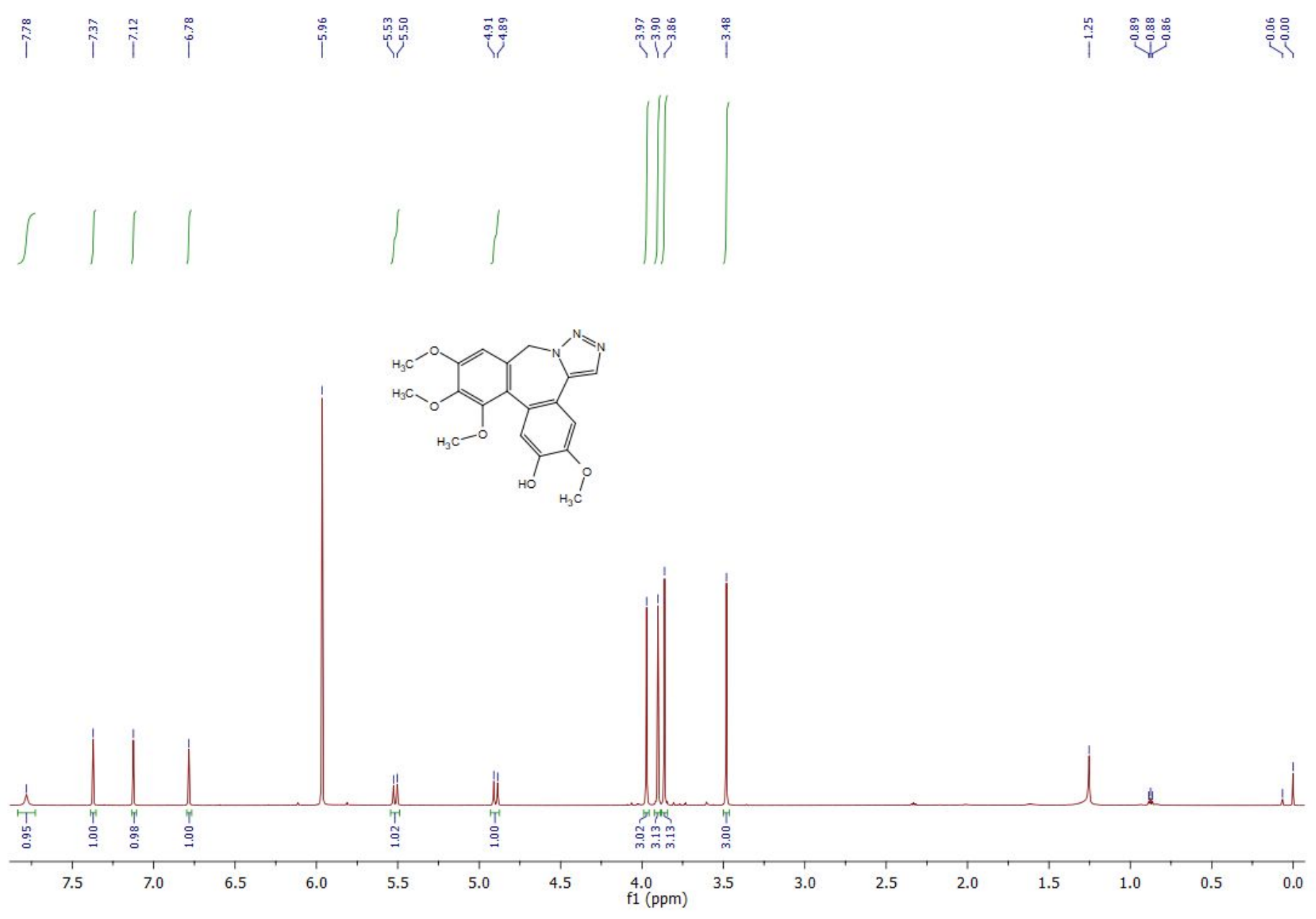


${ }^{13}$ C NMR Spectra of (6a) (600 MHz, $\left.\mathrm{C}_{2} \mathrm{D}_{2} \mathrm{Cl}_{4}\right)$ :

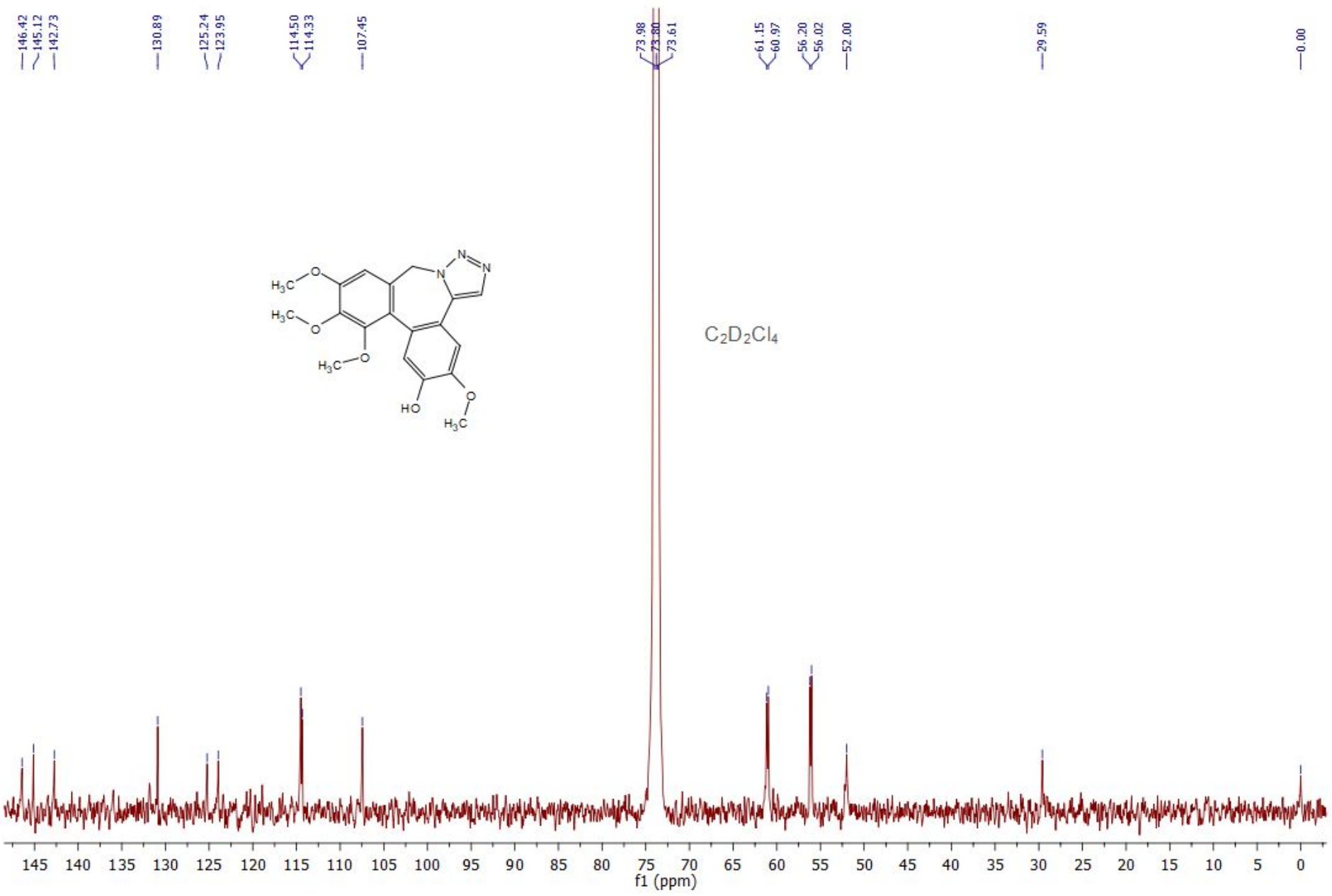


${ }^{\mathbf{1}} \mathbf{H}$ NMR Spectra of (7a) $\left(300 \mathrm{MHz}, \mathrm{CDCl}_{3}\right)$ :

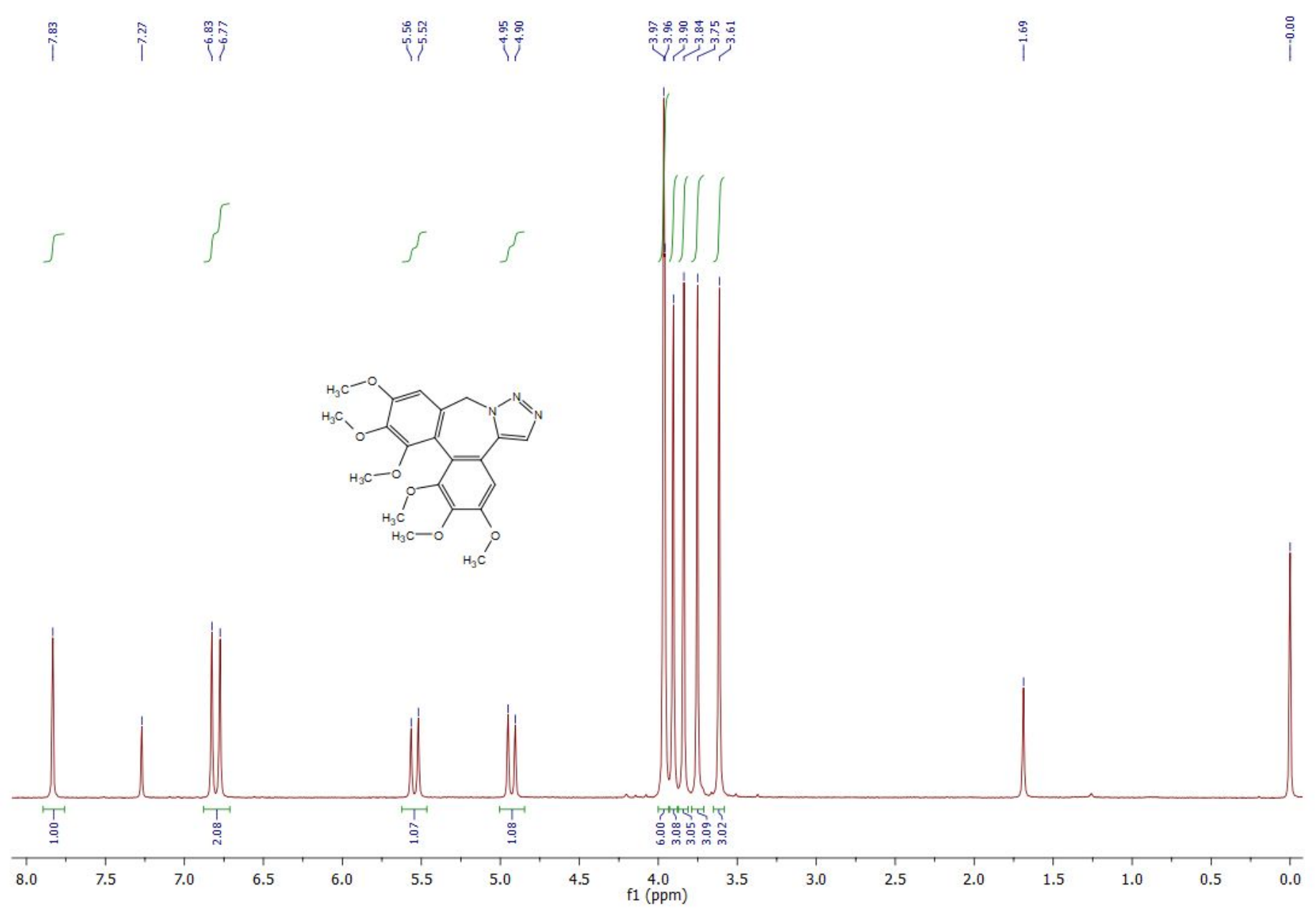


${ }^{13}$ C NMR Spectra of (7a) (300 MHz, $\left.\mathrm{CDCl}_{3}\right)$ :

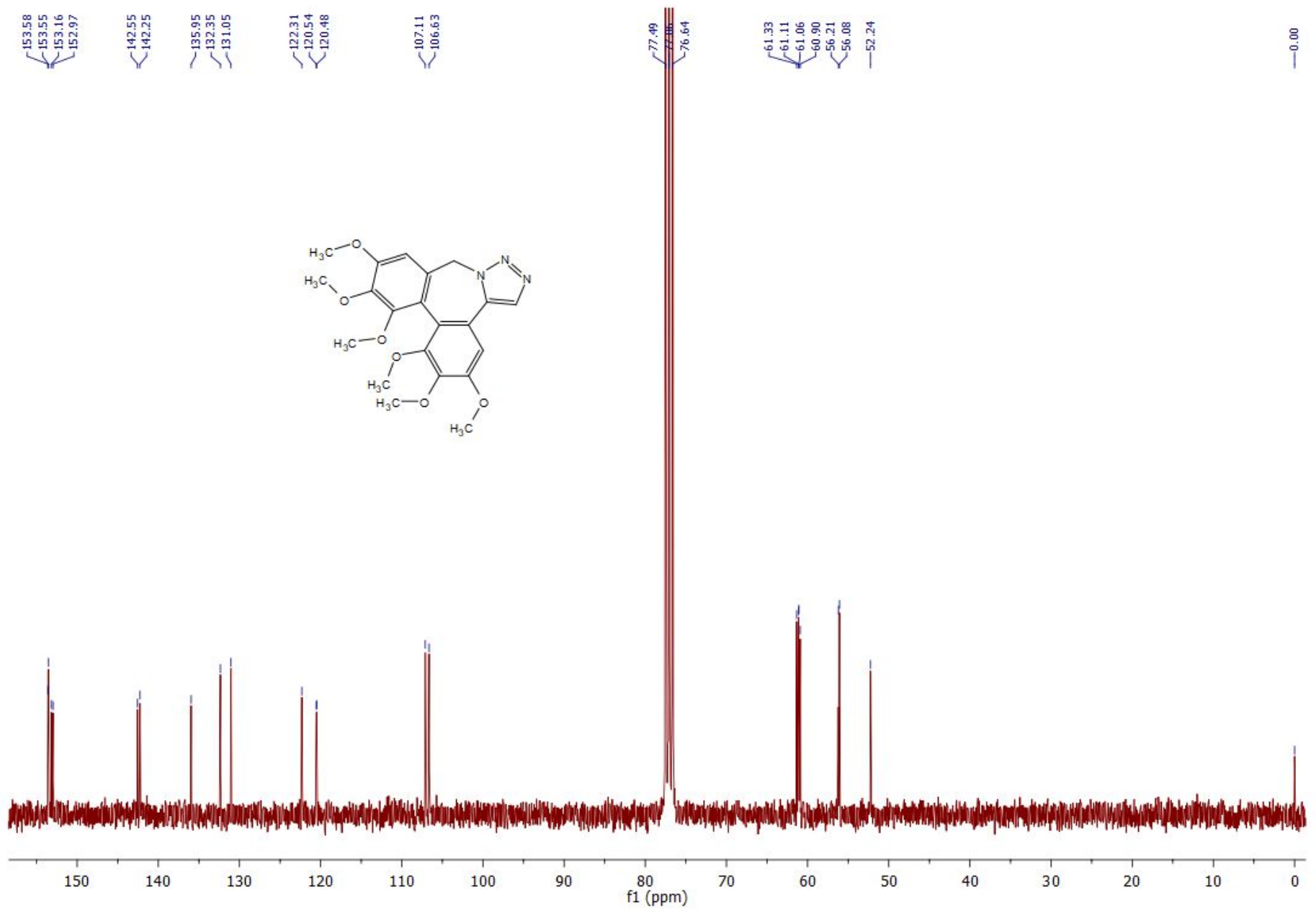


${ }^{\mathbf{1}} \mathbf{H}$ NMR Spectra of (8a) (300 MHz, $\left.\mathrm{CDCl}_{3}\right)$ :
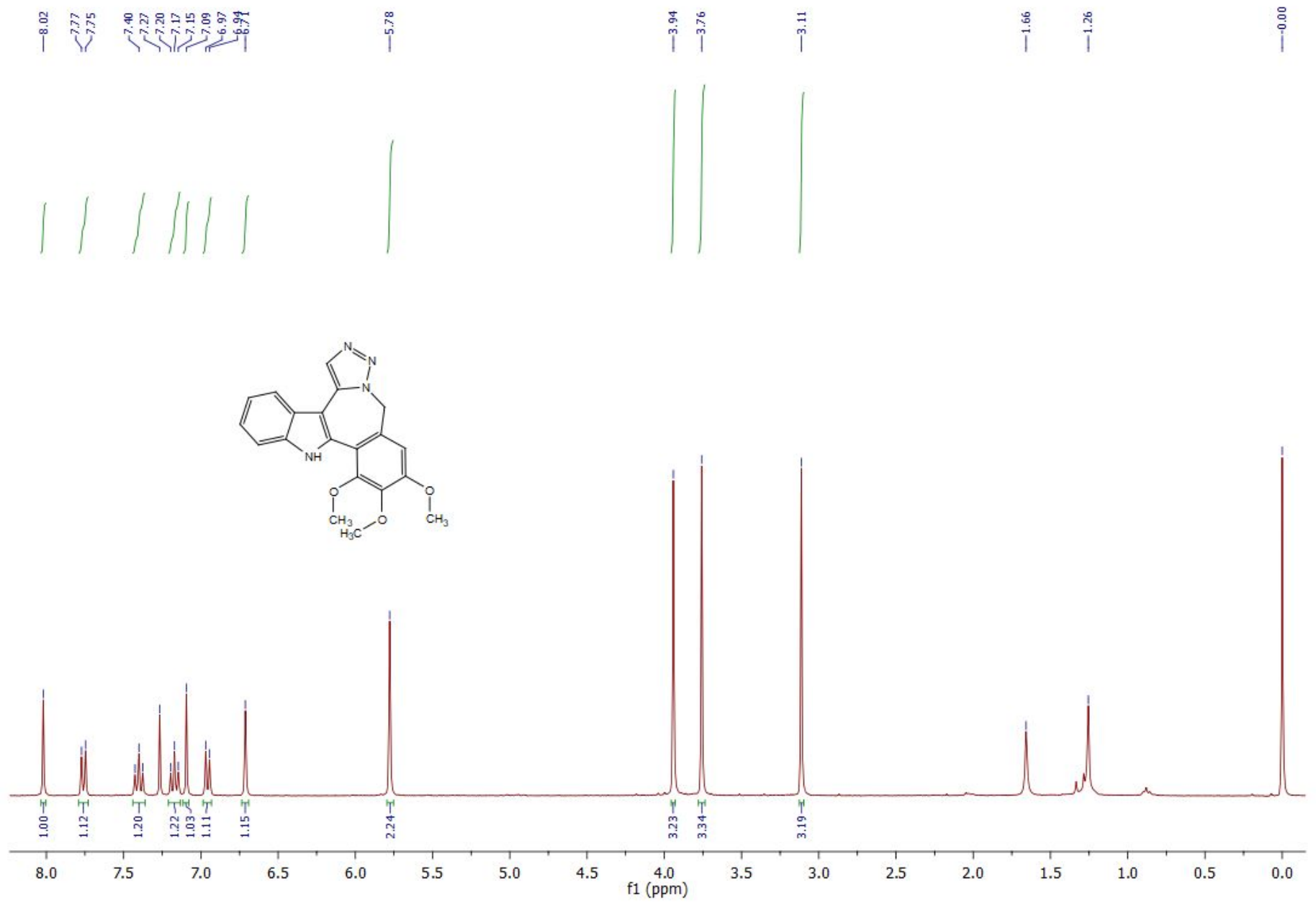
${ }^{13}$ C NMR Spectra of (8a) (600 MHz, $\left.\mathrm{CDCl}_{3}\right)$ :

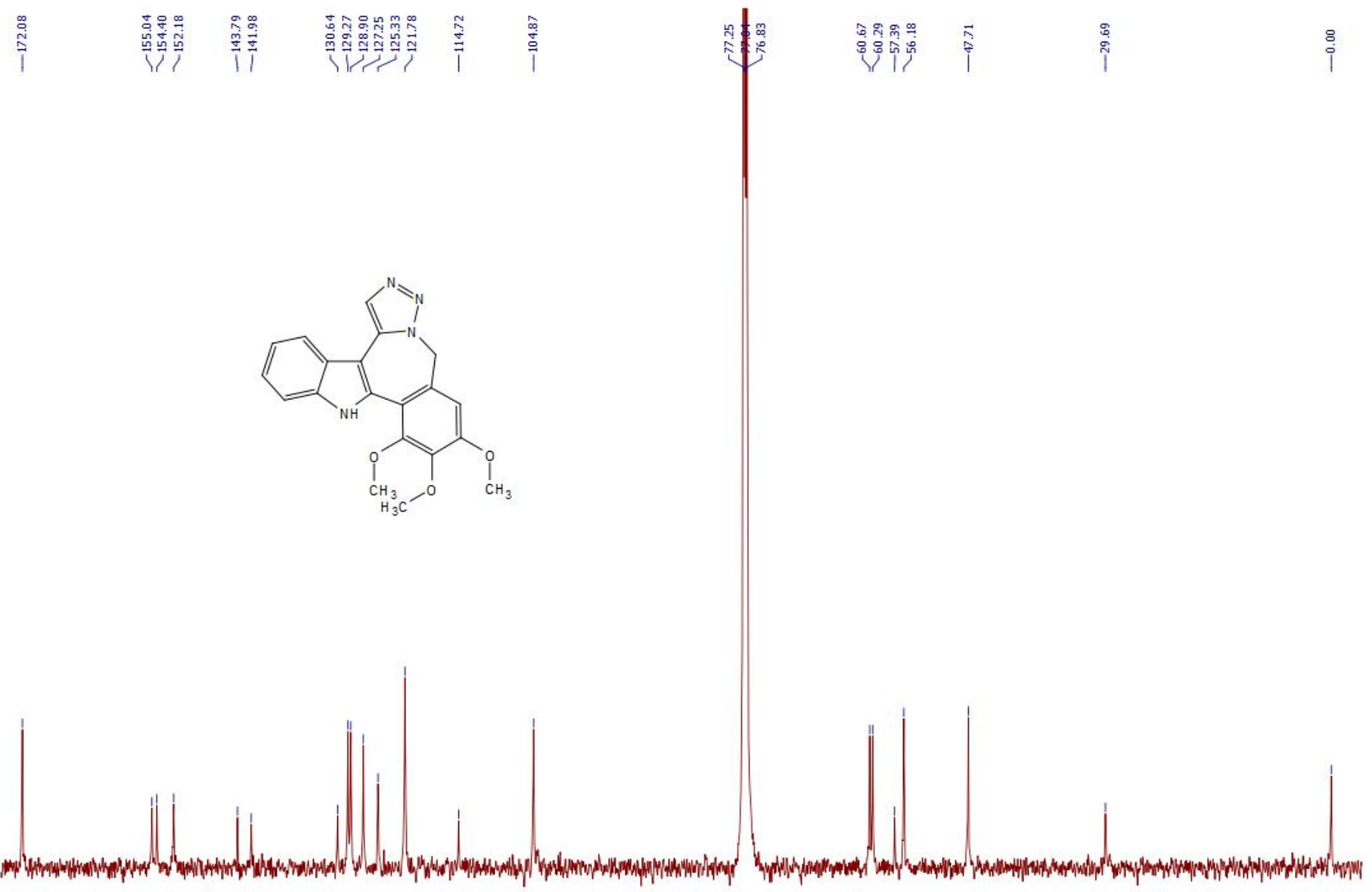

\begin{tabular}{|c|c|c|c|c|c|c|c|c|c|c|c|c|c|c|c|c|}
\hline 1 & 1 & 1 & 1 & 1 & 1 & 1 & 1 & 1 & 1 & 1 & 1 & 1 & 1 & 1 & 1 & 1 \\
\hline 170 & 160 & 150 & 140 & 130 & 120 & 110 & 100 & ${ }_{\mathrm{f} 1(\mathrm{ppm})}^{80}$ & 70 & 60 & 50 & 40 & 30 & 20 & 10 & 0 \\
\hline
\end{tabular}


${ }^{\mathbf{1}} \mathbf{H}$ NMR Spectra of (9a) (300 MHz, $\left.\mathrm{CDCl}_{3}\right)$ :

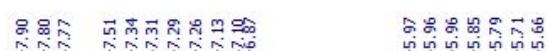

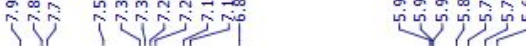

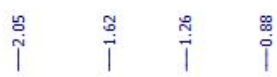
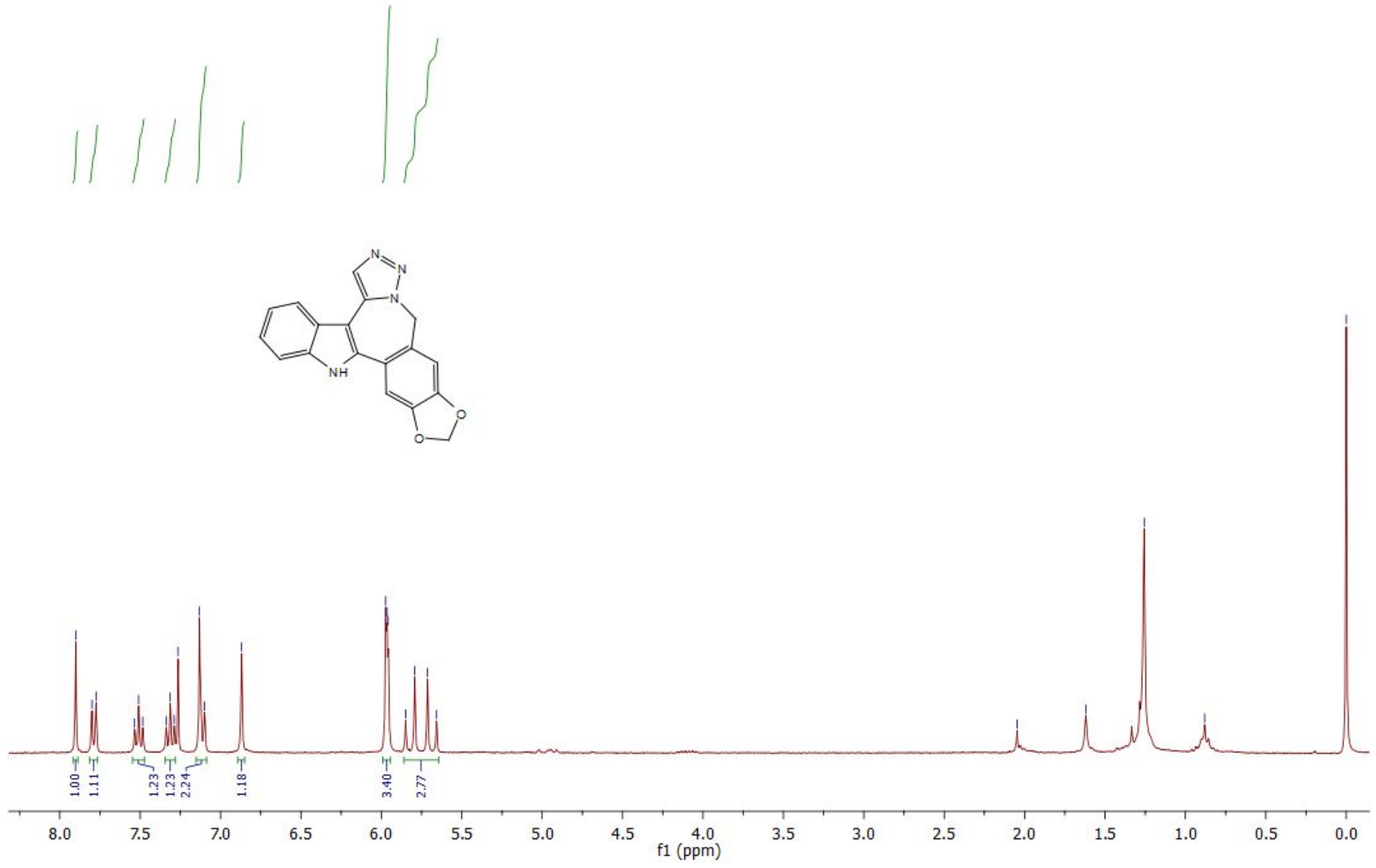
${ }^{13}$ C NMR Spectra of (9a) (600 MHz, $\left.\mathrm{CDCl}_{3}\right)$ :

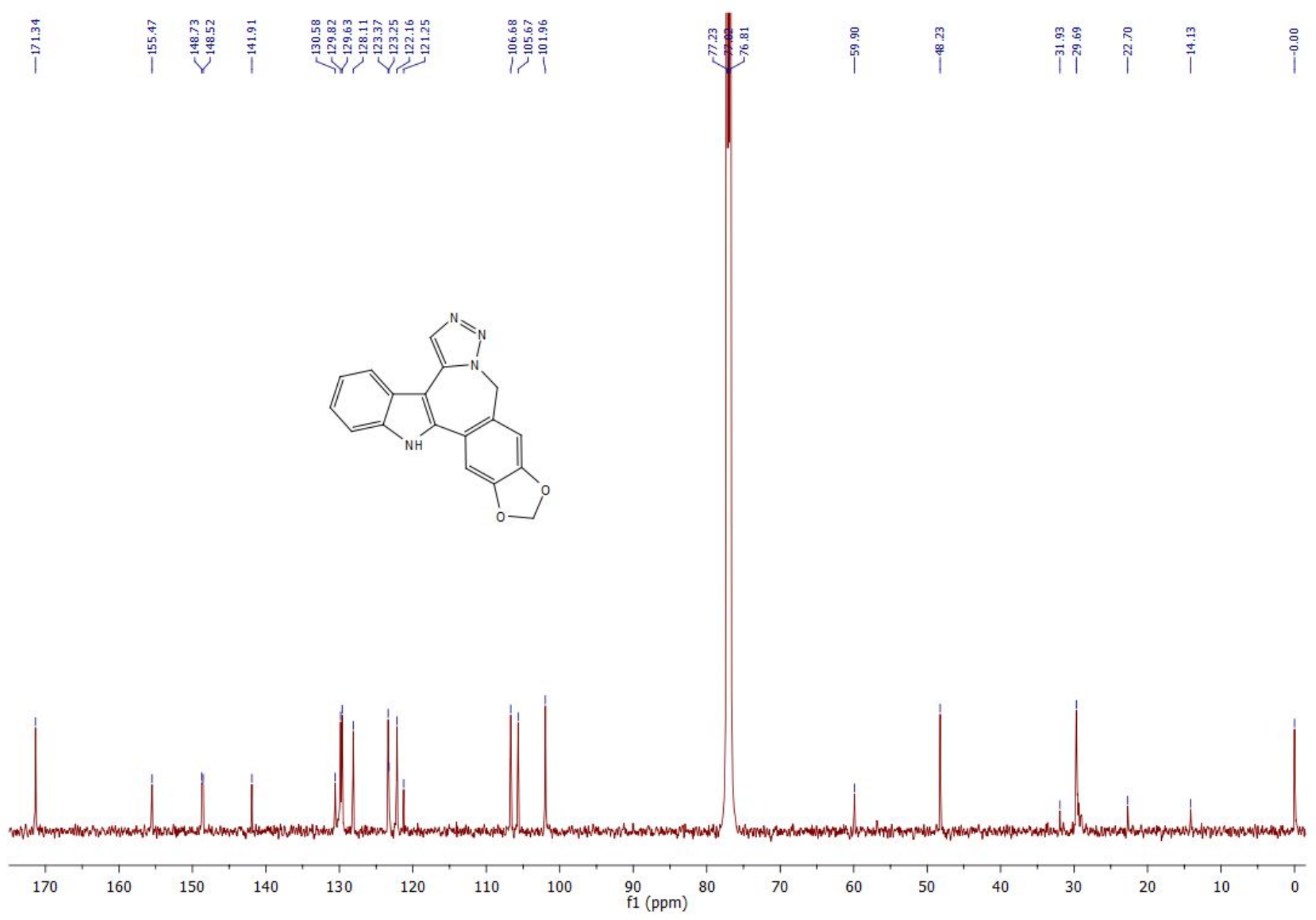


${ }^{1}$ H NMR Spectra of (10a) (300 MHz, $\left.\mathrm{CDCl}_{3}\right)$ :

ホ

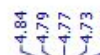

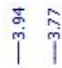

$\stackrel{8}{i}$

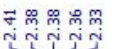

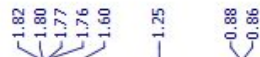

$\stackrel{0}{i}$
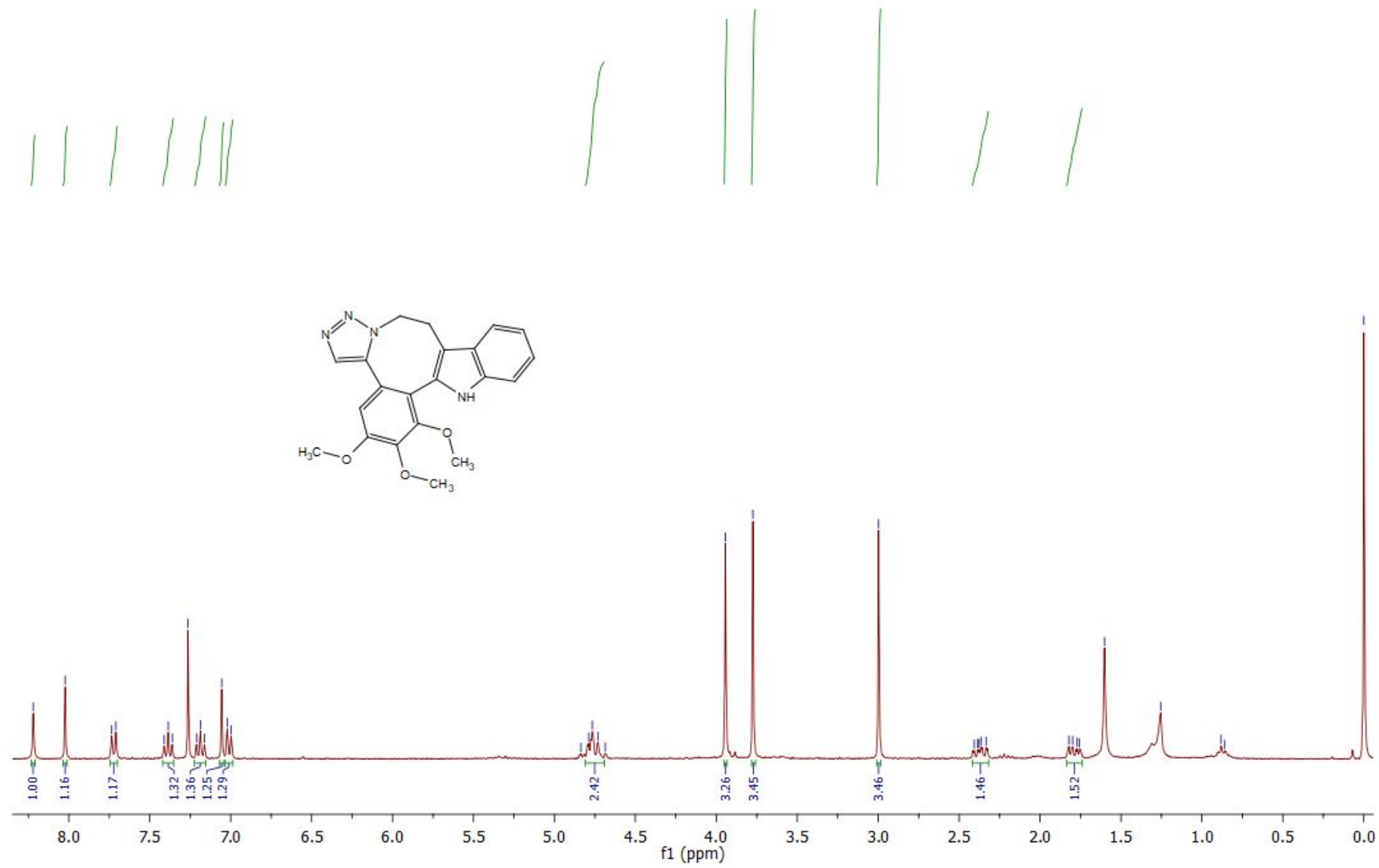
${ }^{13}$ C NMR Spectra of (10a) (600 MHz, $\left.\mathrm{CDCl}_{3}\right)$ :
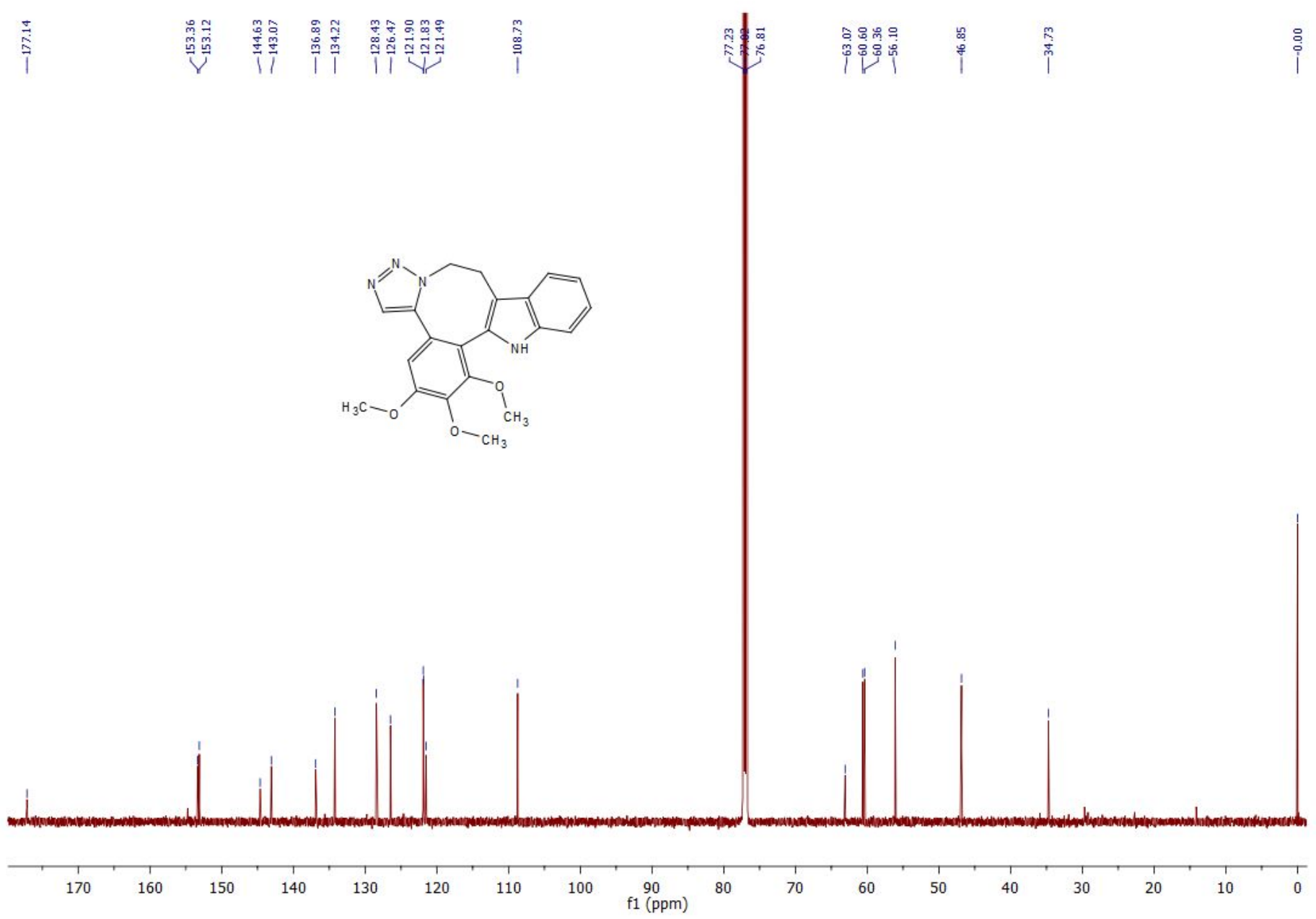
${ }^{\mathbf{1}} \mathbf{H}$ NMR Spectra of (11ab) (300 MHz, $\left.\mathrm{CDCl}_{3}\right)$ :

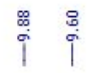

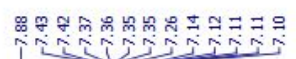

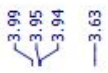

贷尗

i.

$\int$

|| ||
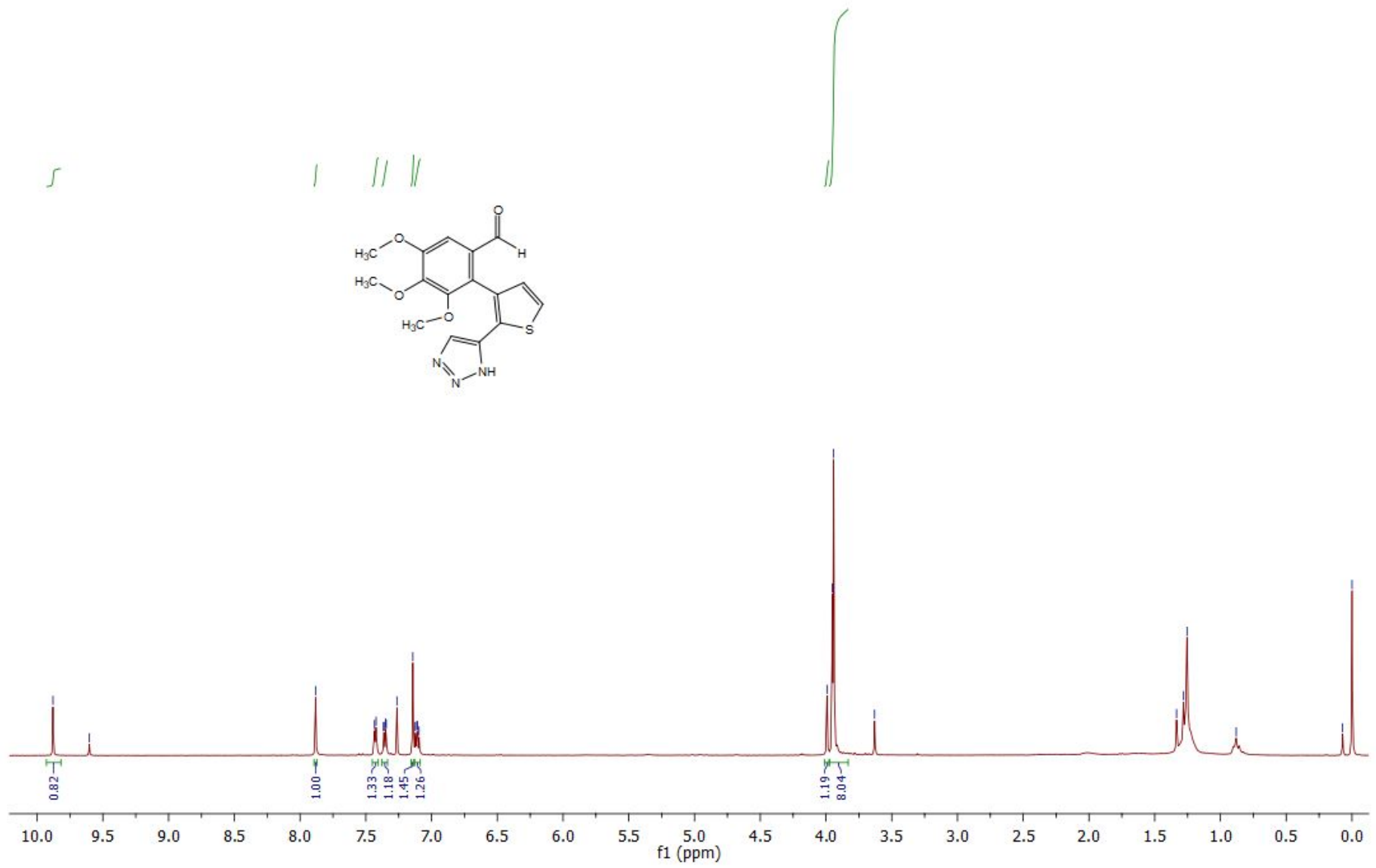
${ }^{13}$ C NMR Spectra of (11ab) (600 MHz, $\left.\mathrm{CDCl}_{3}\right)$ :

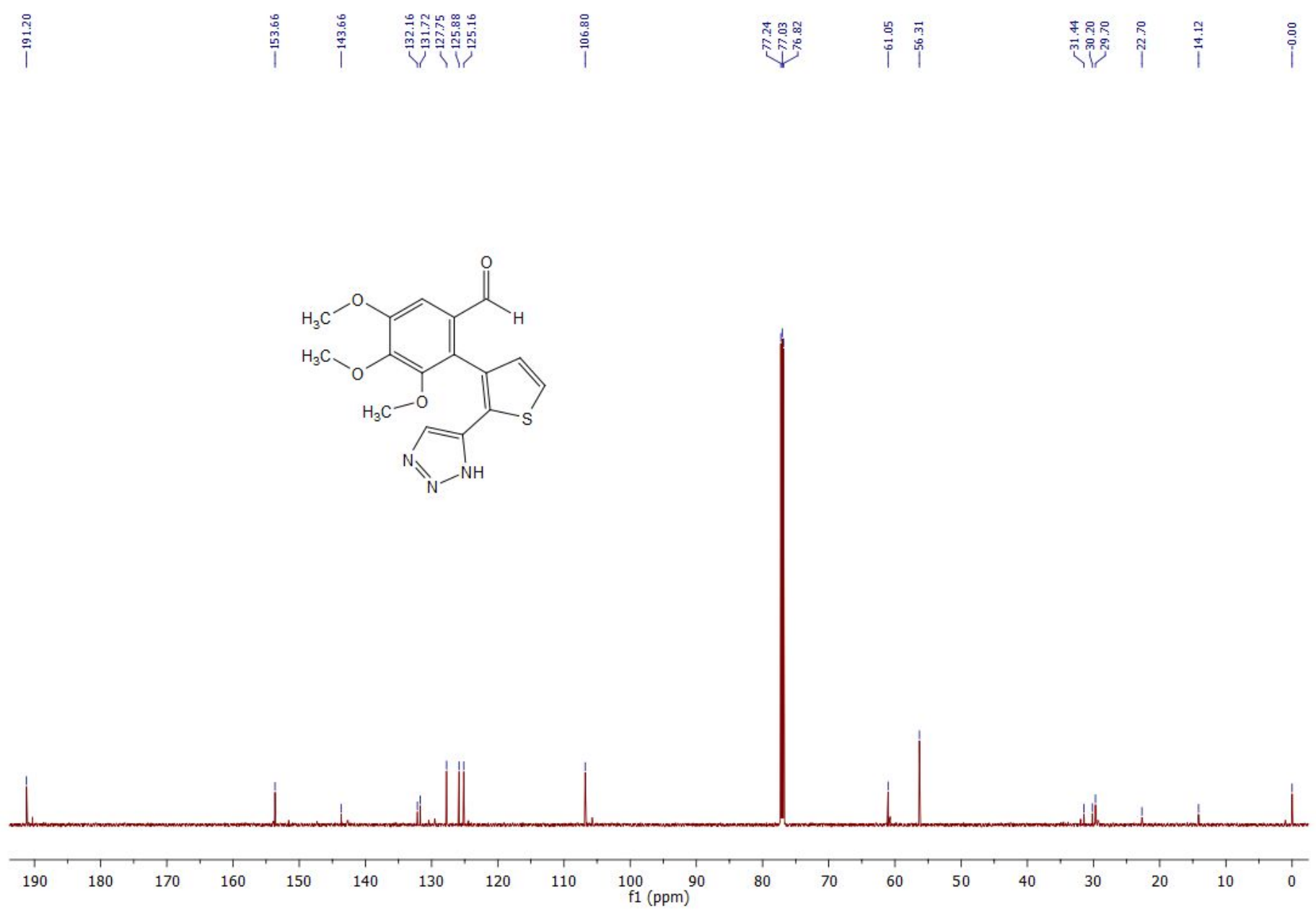

